\title{
K-PROPERTY FOR MAHARAM EXTENSIONS OF NONSINGULAR BERNOULLI AND MARKOV SHIFTS
}

\author{
Alexandre I. Danilenko and Mariusz LemańcZyK
}

\begin{abstract}
It is shown that each conservative nonsingular Bernoulli shift is either of type $I I_{1}$ or $I I I_{1}$. Moreover, in the latter case the corresponding Maharam extension of the shift is a $K$-automorphism. This extends earlier results obtained by Z. Kosloff for the equilibrial shifts. Nonequilibrial shifts of type $I I I_{1}$ are constructed. We further generalize (partly) the main results to nonsingular Markov shifts.
\end{abstract}

\section{INTRODUCTION}

In this paper we study asymptotic properties of nonsingular Bernoulli and nonsingular Markov shifts. By a nonsingular Bernoulli shift we mean the 2 -sided shift $\widetilde{T}$ on the infinite product space $\widetilde{X}:=\{0,1\}^{\mathbb{Z}}$ equipped with the infinite product measure $\bigotimes_{i \leq 0} \mu_{1} \otimes \bigotimes_{i>1} \mu_{i}$, where the probability measures $\mu_{i}, i \geq 1$, are chosen in such a way that the shift is non-singular. We call the Bernoulli shift equilibrial if $\mu_{1}(0)=\mu_{1}(1)=0.5$. We are interested in nonsingular Bernoulli shifts not admitting finite invariant equivalent measures. It is easy to construct dissipative (in fact, totally dissipative) nonsingular Bernoulli shifts. The first example of a conservative nonsingular Bernoulli shift not admitting a finite invariant equivalent measure was constructed by Krengel in $1970[\mathrm{Kr}]$. Later Hamachi presented another family of conservative nonsingular Bernoulli shifts of type $I I I$, i.e. transformations which have no $\sigma$-finite invariant equivalent measures, neither finite nor infinite [Ha]. This was even more refined in [Ko1], where Kosloff constructed a family of nonsingular Bernoulli shifts of type $I I I_{1}$. This means that the Maharam extension of the shift is ergodic. In a subsequent paper [Ko2] he showed that each conservative nonsingular equilibrial Bernoulli shift either admits a finite invariant equivalent measure or is of type $I I I_{1}$. Moreover, in the latter case the corresponding Maharam extension of the shift has property $K$ (in the sense of Silva and Thieullen [SiTh]). This implies that the aforementioned Bernoulli shifts from $[\mathrm{Kr}]$ and $[\mathrm{Ha}]$ are all of type $I I I_{1}$.

Only equilibrial Bernoulli shifts were considered in [Kr], [Ha], [Ko1] and [Ko2]. In the first part of the present paper we extend the main results of [Ko2] to the general nonsingular Bernoulli shifts:

Research of the second named author is supported in part by Narodowe Centrum Nauki UMO2014/15/B/ST1/03736.

Research of the two authors is supported in part by the special program of invitations of the semester "Ergodic Theory and Dynamical Systems in their Interactions with Arithmetic and Combinatorics", Chair Jean Morlet, 1.08.2016-30.01.2017. 


\section{Theorem 3.1 and Corollary 3.3.}

(i) If $\sum_{n=1}^{\infty}\left(\mu_{n}(0)-\mu_{1}(0)\right)^{2}<\infty$ then $\mu$ is equivalent to $\bigotimes_{n=-\infty}^{\infty} \mu_{1}$ and hence $\widetilde{T}$ is of type $I I_{1}$.

(ii) If $\sum_{n=1}^{\infty}\left(\mu_{n}(0)-\mu_{1}(0)\right)^{2}=\infty$ and $\widetilde{T}$ is conservative then $\widetilde{T}$ is ergodic of type $I I I_{1}$ and the Maharam extension of $\widetilde{T}$ is a weakly mixing $K$-automorphism.

The main difference between the general case and the equilibrial one is that in the general case the cocycle generated by the Radon-Nikodym derivative of a onesided Bernoulli shift and the Radon-Nikodym cocycle of the tail equivalence relation generated by the shift do not coincide (see the proof of Theorem 3.1). Hence we can not apply the Araki-Woods lemma on the Krieger's type of the ITPFI factors [ArWo] which played a crucial role in the equilibrial case. That is why we need first to establish a stronger version of the Araki-Woods lemma (see Proposition 1.5).

We also give a simple explicit inductive construction of the sequence of measures $\left(\mu_{i}\right)_{i=1}^{\infty}$ with an arbitrary $\mu_{1}$ such that the corresponding Bernoulli shift is nonsingular, conservative and not of type $I I_{1}$ (see Theorem 4.2). The conservativeness is the key property to establish. We achieve it by approximating the shift with a sequence of Bernoulli type $I I_{1}$ shifts each of which is, of course, conservative. We do the approximation in such a way that $\widetilde{T}$ inherits conservativeness partly, i.e. on a finite subalgebra of cylinders, from each of these prelimit transformations. In the limit, the subalgebras generate the entire Borel $\sigma$-algebra. Hence $\widetilde{T}$ is conservative.

In the second part of the paper we consider nonsingular Markov shifts, i.e. 2sided shifts on $\widetilde{X}$ equipped with Markov measure $\widetilde{\mu}$ determined by a probability $\lambda$ on $\{0,1\}$ and a sequence of stochastic matrices $\left(P^{(n)}\right)_{n=1}^{\infty}$ (see Sections 5 and 7 ). In the bistochastic case we prove the following analogue of Theorem 3.1.

Theorem 8.1. Let the matrices $P^{(n)}, n \geq 1$, be all bistochastic, $\lambda(0)=\lambda(1)=0.5$ and $P^{(1)}=\left(\begin{array}{ll}0.5 & 0.5 \\ 0.5 & 0.5\end{array}\right)$. If $(\widetilde{X}, \widetilde{\mu}, \widetilde{T})$ is conservative then it is weakly mixing and either of type $I I_{1}$ (if $\left.\sum_{n \geq 1}\left(P_{0,0}^{(n)}-0.5\right)^{2}<\infty\right)$ or of type $I I I_{1}$ (otherwise). In the latter case, the Maharam extension of $(\widetilde{X}, \widetilde{\mu}, \widetilde{T})$ is a $K$-automorphism.

We also prove some partial analogues of Theorem 3.1 in the general (not only bistochastic) Markov case in Theorems 7.5 and 9.4.

The outline of the paper is as follows. In Section 1 we first briefly remind the basic concepts of measurable orbit theory: nonsingular equivalence relation, skewproduct extension, essential value of a cocycle, Krieger's type of an equivalence relation, etc. Then we prove some generalizations of Araki-Woods lemma (see Proposition 1.5, Remarks 1.6 and 1.7) that will be utilized in the later sections. In Section 2 we review the general theory of nonsingular endomorphisms and its relation to the measurable orbit theory. We collect there some facts about conservativeness, recurrence, ergodicity, exactness, Maharam extension and natural extension for endomorphisms. In Section 3 we prove one of the main results of the paper. The Maharam extension of a conservative nonsingular Bernoulli shift (which is the natural extension of a one-sided nonsingular Bernoulli shift admitting no equivalent invariant probability measure) is a $K$-automorphism (Theorem 3.1 ). In Section 4 we give concrete examples of conservative nonsingular Bernoulli shifts of type $I I I_{1}$. In Section 5 we introduce Markov measures on $\{0,1\}^{\mathbb{Z}}$. Some analogues of Kakutani theorem on equivalence of infinite product measures and Kolmogorov 
zero-one law for the Markov measures are under discussion there. In Section 6 we compute Krieger's type of the tail equivalence relation equipped with a stationary Markov measure. In Section 7 we introduce nonsingular one-sided Markov shifts and describe their natural extensions. We find a sufficient condition for the natural extensions of the shifts to be $K$-automorphisms (Theorem 7.5). A necessary condition for conservativeness of the natural extensions is also found (Lemma 7.6). In Section 8 we consider bistochastic nonsingular Markov shifts. We show how to extend the main results obtained in Section 3 for Bernoulli shifts to the bistochastic Markov shifts under some "initial conditions" (Theorem 8.1). The general Markov case is considered in Section 9 (see Theorem 9.4). Section 10 is a list of open problems and remarks related to the subject of the paper.

\section{Measurable equivalence Relations and their CoCyCles. Generalizations of Araki-Woods lemma}

Let $(X, \mathfrak{B}, \mu)$ be a standard $\sigma$-finite measure space. A Borel equivalence relation $\mathcal{R} \subset X \times X$ is called countable if for each $x \in X$, the $\mathcal{R}$-equivalence class $\mathcal{R}(x)$ is countable. $\mathcal{R}$ is called $\mu$-nonsingular if for each subset $A \in \mathfrak{B}$ of zero measure, the subset $\mathcal{R}(A):=\bigcup_{x \in A} \mathcal{R}(x)$ is also of zero measure. If for each $A \in \mathfrak{B}$ of positive measure the intersection $(\mathcal{R}(x) \backslash\{x\}) \cap A$ is nontrivial for a.e. $x \in A$ then $\mathcal{R}$ is called $\mu$-conservative. If the $\sigma$-algebra of $\mathcal{R}$-invariant (i.e. $\mathcal{R}$-saturated) Borel subsets in $X$ is trivial $(\bmod \mu)$ then $\mathcal{R}$ is called $\mu$-ergodic.

From now on we will assume that $\mathcal{R}$ is countable and $\mu$-nonsingular. Given a locally compact second countable group $G$, a Borel map $\alpha: \mathcal{R} \rightarrow G$ is called a cocycle of $\mathcal{R}$ if there is a $\mu$-conull subset $A \subset S$ such that $\alpha(x, y) \alpha(y, z)=\alpha(x, z)$ for all $x, y, z \in A$ such that $(x, y),(y, z) \in \mathcal{R}$. If $\lambda_{G}$ is a left Haar measure on $G$ then we can define the $\alpha$-skew product equivalence relation $\mathcal{R}(\alpha)$ on the product space $\left(X \times G, \mu \times \lambda_{G}\right)$ by setting $(x, g) \sim(y, h)$ if $(x, y) \in \mathcal{R}$ and $h=\alpha(x, y) g$. Of course, $\mathcal{R}(\alpha)$ is countable and $\left(\mu \times \lambda_{G}\right)$-nonsingular. If $\mathcal{R}(\alpha)$ is conservative then $\alpha$ is called recurrent. If $\mathcal{R}(\alpha)$ is ergodic then $\alpha$ is called ergodic. Two cocycles $\alpha, \beta: \mathcal{R} \rightarrow G$ are called cohomologous if there is a Borel map $\phi: X \rightarrow G$ such that $\alpha(x, y)=\phi(x)^{-1} \beta(x, y) \phi(y)$ for all $(x, y) \in \mathcal{R} \cap(B \times B)$, where $B \subset X$ is a $\mu$-conull subset. A cocycle is called a coboundary if it is cohomologous to the trivial cocycle.

The Radon-Nikodym cocycle $\Delta_{\mathcal{R}, \mu}: \mathcal{R} \rightarrow \mathbb{R}_{+}^{*}$ (of the pair $(\mathcal{R}, \mu)$ ) can be defined in several ways. We consider only the following one. Let $\Gamma$ be a countable group of Borel bijections of $X$ such that $\mathcal{R}(x)=\{\gamma x \mid \gamma \in \Gamma\}$ for each $x \in X$. It exists (but is non-unique) according to [FeMo]. We now set

$$
\Delta_{\mathcal{R}, \mu}(x, \gamma x):=(d \mu \circ \gamma / d \mu)(x), \quad x \in X .
$$

Then $\Delta_{\mathcal{R}, \mu}$ is well defined (does not depend on the particular choice of $\Gamma$ ). If $\nu$ is a $\sigma$-finite measure on $X$ which is equivalent to $\mu$ then $\Delta_{\mathcal{R}, \mu}$ is cohomologous to $\Delta_{\mathcal{R}, \nu}$. Conversely, a cocycle of $\mathcal{R}$ which is cohomologous to $\Delta_{\mathcal{R}, \mu}$ is $\Delta_{\mathcal{R}, \nu}$ for a measure $\nu$ equivalent to $\mu$. By the Maharam theorem, $\mathcal{R}$ is $\mu$-conservative if and only if $\Delta_{\mathcal{R}, \mu}$ is recurrent [Sc]. We say that $\mu$ is $\mathcal{R}$-invariant if $\Gamma$ preserves $\mu$ (this does not depend on the particular choice of $\Gamma$ ).

Suppose that $\mathcal{R}$ is $\mu$-ergodic. Given a cocycle $\alpha$ of $\mathcal{R}$ with values in an Abelian group $G$, an element $g \in G$ is called an essential value of $\alpha$ if for each set $A \in \mathfrak{B}$ of positive measure and each neighborhood $U$ of $g$ in $G$, there is a subset $B \subset A$ of positive measure and a one-to-one mapping $\gamma: B \rightarrow A$ such that $(x, \gamma x) \in \mathcal{R}$ and 
$\alpha(x, \gamma x) \in U$ for each $x \in B$. The set of all essential values of $\alpha$ is denoted by $r(\alpha)$. It is a closed subgroup of $G$. If a cocycle $\beta: \mathcal{R} \rightarrow G$ is cohomologous to $\alpha$ then $r(\alpha)=r(\beta)$. The cocycle $\alpha$ is ergodic if and only if $r(\alpha)=G$ [Sc]. It is easy to verify that given another Abelian locally compact second countable group $H$ and a homomorphism $\theta: G \rightarrow H$, then $\theta(r(\alpha)) \subset r(\theta \circ \alpha)$.

In order to verify that an element of $G$ is an essential value of $\alpha$ we will use the following approximation lemma.

Lemma 1.1 (cf. [Ch-Pr, Lemma 2.1]). Let $\mathfrak{A} \subset \mathfrak{B}$ be a semiring such that the corresponding ring $\mathfrak{F}(\mathfrak{A})$ is dense in $\mathfrak{B}$. Let $1>\delta>0$ and let $g \in G$. If for each set $A \in \mathfrak{A}$ of positive measure and a neighborhood $U$ of $g$ there is a subset $B \subset A$ and a one-to-one mapping $\gamma: B \rightarrow A$ such that $\mu(B)>\delta \mu(A),(x, \gamma x) \in \mathcal{R}, \alpha(x, \gamma x) \in U$ and $\delta<\Delta_{\mathcal{R}, \mu}(x, \gamma x)<\delta^{-1}$ for all $x \in B$ then $g \in r(\alpha)$.

Suppose that $\mu$ is non-atomic and $\mathcal{R}$ is $\mu$-ergodic. Then $\mathcal{R}$ is called of type $I I$ if there is a $\sigma$-finite $\mathcal{R}$-invariant measure $\nu$ equivalent to $\mu$ or, equivalently, $\Delta_{\mathcal{R}, \mu}$ is a coboundary. If, moreover, $\nu(X)<\infty$ then $\mathcal{R}$ is called of type $I I_{1}$. If $\nu(X)=\infty$ then $\mathcal{R}$ is called of type $I I_{\infty}$. If $\mathcal{R}$ is not of type $I I$ then $\mathcal{R}$ is called of type $I I I$. The type $I I I$ admits further classification into subtypes $I I I_{\lambda}, 0 \leq \lambda \leq 1$. If $\Delta_{\mathcal{R}, \mu}$ is ergodic, i.e. $r\left(\Delta_{\mathcal{R}, \mu}\right)=\mathbb{R}_{+}^{*}$, then $\mathcal{R}$ is called of type $I I I_{1}$. If there is $\lambda \in(0,1)$ such that $\Delta_{\mathcal{R}, \mu}$ is cohomologous to a cocycle $\beta$ taking values in the closed subgroup $\left\{\lambda^{n} \mid n \in \mathbb{Z}\right\} \subset \mathbb{R}_{+}^{*}$ and $\beta$ is ergodic as a cocycle with values in this subgroup then $\mathcal{R}$ is called of type $I I I_{\lambda}$. Finally, if $\mathcal{R}$ is if type $I I I$ but not of type $I I I_{\lambda}, 0<\lambda \leq 1$, then $\mathcal{R}$ is called of type $I I I_{0}$. Equivalently, $\mathcal{R}$ is of type $I I I_{0}$ if $\mathcal{R}$ is of type $I I I$ and $\Delta_{\mathcal{R}, \mu}=\{1\}$ (we refer to [FeMo] and [HaOs1] for details).

We recall an easy classic lemma on the type of direct product of two ergodic equivalence relations.

Lemma 1.2 ([ArWo], [HaOs1]). Let $\mathcal{R}_{i}$ be an ergodic $\mu_{i}$-nonsingular equivalence relations on a standard measure space $\left(X_{i}, \mathfrak{B}_{i}, \mu_{i}\right), i=1,2$.

(i) If $\mathcal{R}_{1}$ is of type $I I_{1}$ then $\mathcal{R}_{1} \times \mathcal{R}_{2}$ is of the same type as $\mathcal{R}_{2}$.

(ii) If $\mathcal{R}_{1}$ is of type $I I I_{1}$ then so is $\mathcal{R}_{1} \times \mathcal{R}_{2}$.

(iii) Let $\mathcal{R}_{1}$ be of type $I I I_{\lambda}$ and let $\mathcal{R}_{2}$ be of type $I I I_{\xi}$ with $0<\lambda, \xi<1$ and let $\Lambda:=\left\{\lambda^{n} \xi^{m} \mid n, m \in \mathbb{Z}\right\}$. If $\Lambda=\left\{\eta^{n} \mid n \in \mathbb{Z}\right\}$ for some $\eta \in(0,1)$ then $\mathcal{R}_{1} \times \mathcal{R}_{2}$ is of type $I I I_{\eta}$. Otherwise $\mathcal{R}_{1} \times \mathcal{R}_{2}$ is of type $I I I_{1}$.

In what follows we will consider infinite product spaces. Let $A$ be a finite set. Then the space $A^{\mathbb{N}}$ endowed with the topology of infinite product of the discrete topologies is a compact metric space. Given $n \leq m$ and a finite sequence $a_{n}, \ldots, a_{m}$ of elements from $A$, we denote by $\left[a_{n}, \ldots, a_{m}\right]_{n}^{m}$ the corresponding cylinder in $A^{\mathbb{N}}$, i.e. the subset $\left\{x=\left(x_{j}\right)_{j=1}^{\infty} \in A^{\mathbb{N}} \mid x_{i}=a_{i}\right.$ whenever $\left.n \leq i \leq m\right\}$. Since the algebra $\mathfrak{K}$ consisting of the finite unions of cylinders is nothing but the algebra of clopen subsets in $A^{\mathbb{N}}$ which is a base of the topology on $A^{\mathbb{N}}$, it follows that $\mathfrak{K}$ is dense in the $\sigma$-algebra of Borel subsets with respect to any measure on $A^{\mathbb{N}}$. The tail equivalence relation on $A^{\mathbb{N}}$ is defined by

$$
\left(x_{i}\right)_{i=1}^{\infty} \sim\left(y_{i}\right)_{i=1}^{\infty} \text { if there is } N>0 \text { such that } x_{i}=y_{i} \text { for all } i \geq N .
$$

Let $(X, \mu)=\left(\{0,1\}^{\mathbb{N}}, \mu_{1} \times \mu_{2} \times \cdots\right)$ such that $\mu_{i}(0)>0, \mu_{i}(1)>0$ and $\mu_{i}(0)+$ $\mu_{i}(1)=1$ for all $i \in \mathbb{N}$. Let $\mathcal{R}$ denote the tail equivalence relation on $X$. It is easy to verify that $\mathcal{R}$ is $\mu$-nonsingular and $\Delta_{\mathcal{R}, \mu}(x, y)=\prod_{i>0} \mu_{i}\left(y_{i}\right) / \mu_{i}\left(x_{i}\right)$ for all pairs 
$(x, y) \in \mathcal{R}, x=\left(x_{i}\right)_{i>0}, y=\left(y_{i}\right)_{i>0}$. According to Kolmogorov zero-one law, $\mathcal{R}$ is $\mu$-ergodic.

Lemma $1.3[\mathrm{ArWo}$. Let $(X, \mu, \mathcal{R})$ be as above and there exist $\lambda \in(0,1]$ and a sequence $\epsilon_{i} \rightarrow 0$ such that

$$
\mu_{i}(0)=\frac{1}{1+\lambda e^{\epsilon_{i}}} \quad \text { and } \quad \mu_{i}(1)=\frac{\lambda e^{\epsilon_{i}}}{1+\lambda e^{\epsilon_{i}}}, \quad i>0
$$

(i) If $\sum_{i>0} \epsilon_{i}^{2}<\infty$ then $\mu$ is equivalent to the infinite product $\kappa_{\lambda} \times \kappa_{\lambda} \times \cdots$, where $\kappa_{\lambda}(0)=1 /(1+\lambda)$ and $\kappa_{\lambda}(1)=\lambda /(1+\lambda)$. Hence $\mathcal{R}$ is of type $I I I_{\lambda}$ in case $0<\lambda<1$ or of type $I I_{1}$ in case $\lambda=1$.

(ii) If $\sum_{i>0} \epsilon_{i}^{2}=\infty$ then $\mathcal{R}$ is of type $I I I_{1}$.

Remark 1.4. The claim $(i)$ of Lemma 1.3 follows straightforwardly from the Kakutani theorem on equivalence of infinite product measures [Ka]. The claim $(i i)$ is more involved. It is a particular case of Lemma 9.3 from the paper [ArWo] devoted to the theory of operator algebras. It was an attempt in [Os] to give a pure measure theoretical proof of this result. However, in our opinion, that proof has a couple of flaws (for instance, in the place where the author applies the central limit theorem). In [BrDo] Brown and Dooley used the language of $G$-measures to provide a new proof of a simple case of Lemma 1.3( $i i)$ (with $\lambda=1$ ). In a subsequent paper [Br-La] Brown, Dooley and Lake showed that this proof is false. They gave another proof (only for the case $\lambda=1$ ) which did not use the $G$-measures [Br-La]. In our opinion, their new proof is somewhat more complicated comparatively with the original argument by Araki and Woods. Below we will need the following proposition whose proof implies Lemma 1.3(ii) (see Remark 1.6). To prove it we use an argument which is close to the argument utilized in [ArWo, Lemma 9.3].

Proposition 1.5. Let $(X, \mu, \mathcal{R})$ be as in Lemma 1.3(ii). Define a cocycle $\Lambda: \mathcal{R} \rightarrow$ $\mathbb{R}$ by setting

$$
\Lambda(x, y)=\sum_{i>0}\left(y_{i}-x_{i}\right) \log \lambda
$$

Then the cocycle $\alpha:=\log \Delta_{\mathcal{R}, \mu}-\Lambda$ of $\mathcal{R}$ with values in $\mathbb{R}$ is ergodic.

Proof. Fix an infinite subset $J \subset \mathbb{N}$ such that $\sum_{i \in J} \epsilon_{i}^{2}<\infty$. Applying Lemma 1.3(i) we replace $\mu$ by an equivalent measure for which (1-1) is satisfied, $\sum_{i>0} \epsilon_{i}^{2}=\infty$ and $\epsilon_{i}=0$ if $i \in J$. Therefore without loss of generality we may think that the triplet $(X, \mu, \mathcal{R})$ is isomorphic to the triplet $(Z, \eta, \mathcal{T})$, where $Z:=(\{0,1\} \times\{0,1\})^{\mathbb{N}}$, $\eta:=\bigotimes_{i=1}^{\infty}\left(\mu_{i} \times \mu_{0}\right), \mu_{0}(0):=1 /(1+\lambda)$ and $\mu_{0}(1):=\lambda /(1+\lambda)$ and $\mathcal{T}$ is the tail equivalence relation on $Z$. It is easy to verify that

$$
\log \Delta_{\mathcal{T}, \eta}\left(z, z^{\prime}\right)=\sum_{i>0}\left(\left(x_{i}^{\prime}-x_{i}\right)\left(\log \lambda+\epsilon_{i}\right)+\left(y_{i}^{\prime}-y_{i}\right) \log \lambda\right)
$$

where $z=\left(x_{i}, y_{i}\right)_{i>0}$ and $z^{\prime}=\left(x_{i}^{\prime}, y_{i}^{\prime}\right)_{i>0}$ are $\mathcal{T}$-equivalent points, and $x_{i}, x_{i}^{\prime}, y_{i}, y_{i}^{\prime} \in$ $\{0,1\}$ for each $i>0$. Computing $\alpha$ in the "new coordinates" we obtain that

$$
\alpha\left(z, z^{\prime}\right)=\sum_{i>0} \epsilon_{i}\left(x_{i}^{\prime}-x_{i}\right) \quad \text { for all }\left(z, z^{\prime}\right) \in \mathcal{T}
$$


Denote by $\tau$ the flip on $\{0,1\} \times\{0,1\}$, i.e. $\tau(i, j)=(j, i)$. Given $z=\left(x_{i}, y_{i}\right)_{i>0} \in Z$ and $n>0$, we denote by $z^{* n}$ the element $\left(x_{i}^{*}, y_{i}^{*}\right)_{i>0} \in Z$, where

$$
\left(x_{i}^{*}, y_{i}^{*}\right):= \begin{cases}\tau\left(x_{i}, y_{i}\right), & \text { for } i=1, \ldots, n \\ \left(x_{i}, y_{i}\right), & \text { otherwise. }\end{cases}
$$

Of course, $\left(z, z^{* n}\right) \in \mathcal{T}$.

Claim 1. For each $a \in \mathbb{R}$,

$$
\lim _{n \rightarrow \infty} \eta\left(\left\{z \in Z \mid \alpha\left(z, z^{* n}\right)>a\right\}\right)=0 .
$$

To prove this claim we define mappings $X_{i}: Z \rightarrow \mathbb{R}$ by

$$
X_{i}(z):= \begin{cases}(-1)^{x_{i}} \epsilon_{i}, & \text { if } x_{i} \neq y_{i} \\ 0, & \text { otherwise. }\end{cases}
$$

Then $X_{1}, X_{2}, \ldots$ is a sequence of independent random variables and $\left|X_{i}\right| \leq 1$ for all $i$. The expected value $E\left(X_{i}\right)$ equals

$$
\epsilon_{i} \mu_{i}(0) \mu_{0}(1)-\epsilon_{i} \mu_{i}(1) \mu_{0}(0)=\frac{\lambda \epsilon_{i}\left(1-e^{\epsilon_{i}}\right)}{(1+\lambda)\left(1+\lambda e^{\epsilon_{i}}\right)}=\frac{-\lambda \epsilon_{i}^{2}}{(1+\lambda)^{2}}(1+\bar{o}(1)) .
$$

In a similar way,

$$
E\left(X_{i}^{2}\right)=\frac{\lambda \epsilon_{i}^{2}\left(1+e^{\epsilon_{i}}\right)}{(1+\lambda)\left(1+\lambda e^{\epsilon_{i}}\right)}=\frac{2 \lambda \epsilon_{i}^{2}}{(1+\lambda)^{2}}(1+\bar{o}(1)) .
$$

Therefore the variance $\sigma^{2}\left(X_{i}\right)$ equals $\frac{2 \lambda \epsilon_{i}^{2}}{(1+\lambda)^{2}}(1+\bar{o}(1))$. Hence $\lim _{i \rightarrow \infty} E\left(X_{i}\right)=0$ and $\sum_{i=1}^{\infty} \sigma^{2}\left(X_{i}\right)=+\infty$. It now follows from the central limit theorem for uniformly bounded sequences of independent random variables that

$$
\frac{\sum_{i=1}^{n}\left(X_{i}-E\left(X_{i}\right)\right)}{\sqrt{\sum_{i=1}^{n} \sigma^{2}\left(X_{i}\right)}} \text { approaches the normal distribution as } n \rightarrow \infty .
$$

We note that

$$
\alpha\left(z, z^{* n}\right)=\sum_{i=1}^{n} \epsilon_{i}\left(y_{i}-x_{i}\right)=\sum_{i=1}^{n} X_{i}(z) .
$$

Let $A_{n}:=\left\{z \in Z \mid \alpha\left(z, z^{* n}\right)>a\right\}$. Since $\sqrt{\sum_{i=1}^{n} \sigma^{2}\left(X_{i}\right)} \rightarrow \infty$ and

$$
-\infty=\lim _{n \rightarrow \infty} \frac{-\sqrt{\lambda} \sum_{i=1}^{n} \epsilon_{i}^{2}}{(1+\lambda) \sqrt{2 \sum_{i=1}^{n} \epsilon_{i}^{2}}}=\lim _{n \rightarrow \infty} \frac{\sum_{i=1}^{n} E\left(X_{i}\right)}{\sqrt{\sum_{i=1}^{n} \sigma^{2}\left(X_{i}\right)}}
$$

it follows that

$$
\min _{z \in A_{n}} \frac{\sum_{i=1}^{n}\left(X_{i}(z)-E\left(X_{i}\right)\right)}{\sqrt{\sum_{i=1}^{n} \sigma^{2}\left(X_{i}\right)}} \rightarrow+\infty
$$

as $n \rightarrow \infty$. In view of (1-4), we obtain that $\eta\left(A_{n}\right) \rightarrow 0$, as claimed.

Claim 2. Fix $r<-1$. We are going to show that $r$ is an essential value of $\alpha$. Let $\epsilon>0$. Choose $k>0$ such that $\epsilon_{i}<\epsilon$ for all $i \geq k$. Fix a cylinder 
$C:=\left[\left(a_{1}, b_{1}\right), \ldots,\left(a_{k}, b_{k}\right)\right]_{1}^{k} \subset Z$. It follows from the proof of Claim 1 that there are $N>0$ and a subset $I \subset(\{0,1\} \times\{0,1\})^{N-k}$ such that for the subset

$$
A:=\bigsqcup_{\left(z_{k+1}, \ldots, z_{N}\right) \in I}\left[\left(a_{1}, b_{1}\right), \ldots,\left(b_{k}, a_{k}\right), z_{k+1}, \ldots, z_{N}\right]_{1}^{N} \subset C,
$$

we have $\eta(A)>0.5 \eta(C)$ and $\max _{z \in A} \alpha\left(z, z^{\bullet N}\right)<r$, where

$$
z^{\bullet l}:=\left(\left(a_{1}, b_{1}\right), \ldots,\left(a_{k}, b_{k}\right), \tau\left(z_{k+1}\right), \ldots, \tau\left(z_{l}\right), z_{l+1}, z_{l+2}, \ldots\right)
$$

and $l=k+1, \ldots, N$. Note that by $(1-3),\left|\alpha\left(z, z^{\bullet l}\right)-\alpha\left(z, z^{\bullet(l+1)}\right)\right| \leq \epsilon_{l+1}$. For $z \in A$, let $l(z)$ be the smallest number $l>k$ such that $\alpha\left(z, z^{\bullet l}\right)<r$. Then $l(z) \leq N$ and $\left|\alpha\left(z, z^{\bullet l(z)}\right)-r\right| \leq \epsilon_{l(z)}<\epsilon$. We now set

$$
\phi(z):=z^{\bullet l(z)}, \quad z \in A
$$

Then $(z, \phi(z)) \in \mathcal{T}$, the mapping $\phi: A \ni z \mapsto \phi(z) \in C$ is one-to-one and $|\alpha(z, \phi(z))-r|<\epsilon$ for all $z \in A$. To show that $\phi$ is one-to-one, we suppose that $\phi(z)=\phi\left(z^{\prime}\right)$ for some $z=\left(z_{i}\right)_{i=1}^{\infty}, z^{\prime}=\left(z_{i}^{\prime}\right)_{i=1}^{\infty} \in A$. If $l(z)=l\left(z^{\prime}\right)$ then obviously $z=z^{\prime}$. Therefore suppose that $l(z)>l\left(z^{\prime}\right)$. Then the equality $\phi(z)=\phi\left(z^{\prime}\right)$ yields that $z_{i}=z_{i}^{\prime}$ if $1 \leq i \leq k$ and $\tau\left(z_{i}\right)=\tau\left(z_{i}^{\prime}\right)$ if $k+1 \leq i \leq l\left(z^{\prime}\right)$. Hence $z_{i}=z_{i}^{\prime}$ whenever $1 \leq i \leq l\left(z^{\prime}\right)$. Therefore $\alpha\left(z, z^{\bullet l\left(z^{\prime}\right)}\right)=\alpha\left(z^{\prime},\left(z^{\prime}\right)^{\bullet l\left(z^{\prime}\right)}\right)<r$, a contradiction which implies $l(z)=l\left(z^{\prime}\right)$ and hence $z=z^{\prime}$. It follows from (1-2) and (1-3) that $\log \Delta_{\mathcal{T}, \eta}(z, \phi(z))=\alpha(z, \phi(z))$ for all $z \in A$. Now Lemma 1.1 yields (put $\left.\delta:=e^{r-\epsilon}\right)$ that $r$ is an essential value of $\alpha$. Since $r$ is an arbitrary real number less than -1 , it follows that $r(\alpha)=\mathbb{R}$, i.e. $\alpha$ is ergodic.

Remark 1.6. In fact, we proved more than claimed in the statement of Proposition 1.5. It was shown indeed that $(r, r)$ is an essential value for the "double cocycle" $\alpha \times \log \Delta_{\mathcal{R}, \mu}: \mathcal{R} \rightarrow \mathbb{R} \times \mathbb{R}$ for each $r \in \mathbb{R}$. It follows that $\log \Delta_{\mathcal{R}, \mu}$ is ergodic, i.e. $\mathcal{R}$ is of type $I I I_{1}$. Thus we obtain a new short proof of Lemma 1.3( $\mathrm{ii}$. Moreover, for each $t, r \in \mathbb{R}$, we have that $(t r,(1-t) r)$ is an essential value of the cocycle $t \alpha \times(1-t) \log \Delta_{\mathcal{R}, \mu}$. This yields that $r$ is is an essential value of the cocycle $\log \Delta_{\mathcal{R}, \mu}-t \Lambda=t \alpha+(1-t) \log \Delta_{\mathcal{R}, \mu}$ of $\mathcal{R}$. We thus obtain that the cocycle $\log \Delta_{\mathcal{R}, \mu}-t \Lambda$ is ergodic for each real $t \in \mathbb{R}$.

Remark 1.7. The group $\Sigma_{0}$ of finite permutations of $\mathbb{N}$ acts naturally on $X$. Denote by $S$ the $\Sigma_{0}$-orbit equivalence relation. Of course, $\mathcal{S}$ is a subrelation of $\mathcal{R}$. It follows from the proof of Proposition 1.5 (see the definition of $\phi$ ) and Remark 1.6 that the restriction of the cocycle $\log \Delta_{\mathcal{R}, \mu}-t \Lambda$ to $\mathcal{S}$ is also ergodic for each real $t \in \mathbb{R}$.

\section{Nonsingular Endomorphisms}

Let $T$ be a countable-to-one $\mu$-nonsingular endomorphism of a $\sigma$-finite standard measure space $(X, \mathfrak{B}, \mu)$. The $\mu$-nonsingularity means that $\mu \circ T^{-1} \sim \mu$. If for each $A \in \mathfrak{B}$ of positive measure there is $n>0$ such that $\mu\left(T^{-n} A \cap A\right)>0$ then $T$ is called conservative. If $T$ is not conservative then $T$ is called dissipative. If $\mu\left(T^{-1} A \triangle A\right)=0$ implies $\mu(A)=0$ or $\mu(X \backslash A)=0$ then $T$ is called ergodic. If $\bigcap_{n>0} T^{-n} \mathfrak{B}=\{\emptyset, X\}$ (modulo the subsets of zero $\mu$-measure) then $T$ is called exact.

The orbit equivalence relation $\mathcal{R}_{T}$ of $T$ is given by the formula $(x, y) \in \mathcal{R}_{T}$ if and only if there are $n, m \geq 0$ such that $T^{n} x=T^{m} y$. Then $\mathcal{R}_{T}$ is $\mu$-nonsingular. We also consider a subrelation $\mathcal{S}_{T}$ of $\mathcal{R}_{T}:(x, y) \in \mathcal{S}_{T}$ if and only if there is $n \geq 0$ such that $T^{n} x=T^{n} y$. Of course, $\mathcal{S}_{T}$ is also $\mu$-nonsingular. We recall some standard facts. 


\section{Lemma 2.1.}

(i) $\mathcal{R}_{T}$ is $\mu$-ergodic if and only if $T$ is ergodic [Haw].

(ii) $\mathcal{S}_{T}$ is $\mu$-ergodic if and only if $T$ is exact [Haw].

(iii) If $T$ is invertible then $\mathcal{R}_{T}$ is $\mu$-conservative if and only if $T$ is conservative ${ }^{1}$.

From now on we will assume that $T$ is aperiodic, i.e. $\mu\left(\left\{x \in X \mid T^{n} x=x\right\}\right)=0$ for each $n>0$. Then for each Borel function $\phi: X \rightarrow G$, there is a unique cocycle $\alpha_{\phi}$ of $\mathcal{R}_{T}$ with values in $G$ such that $\alpha_{\phi}(x, T x)=\phi(x)$. By $T_{\phi}$ we denote the corresponding skew product transformation of $\left(X \times G, \mu \times \lambda_{G}\right)$ :

$$
T_{\phi}(x, g)=(T x, \phi(x) g) .
$$

Of course, $T_{\phi}$ is a nonsingular countable-to-one endomorphism of $\left(X \times G, \mu \times \lambda_{G}\right)$. It is straightforward to verify that $\mathcal{R}_{T_{\phi}}=\mathcal{R}_{T}\left(\alpha_{\phi}\right)$ and $\mathcal{S}_{T_{\phi}}=\mathcal{S}_{T}\left(\alpha_{\phi}\left\lceil\mathcal{S}_{T}\right)\right.$.

If $T$ is invertible we denote by $\omega_{T, \mu}$ the Radon-Nikodym derivative $d \mu \circ T / d \mu$ : $X \rightarrow \mathbb{R}_{+}^{*}$ of $T$. Then, of course, we have that $\alpha_{\omega_{T, \mu}}=\Delta_{\mathcal{R}_{T}, \mu}$. This definition extends naturally to the general (non-invertible) case as follows. Suppose that $\mu$ is $\sigma$-finite on the $\sigma$-algebra $T^{-1} \mathfrak{B}$. Then by the Radon-Nikodym derivative of $T$ we mean the function $\omega_{T, \mu}:=\left(d \mu / d \mu \circ T^{-1}\right) \circ T$. However, in the non-invertible case we no longer have that $\alpha_{\omega_{T, \mu}}=\Delta_{\mathcal{R}_{T}, \mu}$. The endomorphism $T_{\omega_{T, \mu}}$ is called the Maharam extension of $T$. Choose a measure $\kappa$ on $\mathbb{R}_{+}^{*}$ equivalent to Lebesgue measure such that $\kappa(a B)=a^{-1} \kappa(B)$ for each Borel subset $B \subset \mathbb{R}_{+}^{*}$ and $a \in \mathbb{R}_{+}^{*}$. We will always assume that space $X \times \mathbb{R}_{+}^{*}$ of the Maharam extension is endowed with the measure $\mu \times \kappa$. Then it is easy to see that $T_{\omega_{T, \mu}}$ preserves this measure.

We can associate a linear operator $U_{T}$ in $L^{2}(X, \mu)$ to $T$ in the following way:

$$
U_{T} f(x):=f(T x) \sqrt{\omega_{T, \mu}(x)}, \quad x \in X .
$$

It is easy to see that $U_{T}$ is an isometry. Hence $U_{T}^{*} U_{T}=I$. If $T$ is invertible then $U_{T}$ is unitary and $U_{T}^{n}=U_{T^{n}}$ for all $n \in \mathbb{Z}^{2}$ A useful spectral condition for conservativeness of invertible endomorphisms was found in [Ko2].

Lemma 2.2 [Ko2, Lemma 2.2]. If $T$ is invertible and $\sum_{n \geq 0}\left\langle U_{T}^{n} 1,1\right\rangle<\infty$ then $T$ is dissipative.

If for each positive Borel function $f: X \rightarrow(0,+\infty)$,

$$
\sum_{n \geq 0} f\left(T^{n} x\right) \alpha_{\omega_{T, \mu}}\left(x, T^{n} x\right)=\infty \quad \text { a.e. }
$$

then $T$ is called $\mu$-recurrent $[\mathrm{Si}]$.

\section{Lemma 2.3.}

(i) $T$ is $\mu$-recurrent if and only if $T_{\omega_{T, \mu}}$ is conservative [Si].

(ii) If $T$ preserves $\mu$ then $T$ is $\mu$-recurrent if and only $T$ is conservative.

(iii) If $T$ is $\mu$-recurrent then $T$ is conservative [Si].

(iv) If $T$ is invertible and conservative then $T$ is $\mu$-recurrent.

$(v)$ If $T$ is non-invertible and conservative then there is a measure $\nu$ equivalent to $\mu$ such that $T$ is not $\nu$-recurrent [EiSi].

(vi) If $\mu(X)<\infty$ then $T$ is $\mu$-recurrent if and only if $\sum_{n \geq 0} \alpha_{\omega_{T, \mu}}\left(x, T^{n} x\right)=$ $\infty$ a.e. [Si].

\footnotetext{
${ }^{1}$ This claim is no longer true in the general case where $T$ is non-invertible.

${ }^{2}$ In the non-invertible case, in general, $U_{T}^{n} \neq U_{T^{n}}$ for $n>1$.
} 
Suppose that $\mu$ is $\sigma$-finite on $T^{-1} \mathfrak{B}$. Then there is a standard $\sigma$-finite measure space $(\widetilde{X}, \widetilde{\mathfrak{B}}, \widetilde{\mu})$, an invertible $\mu$-nonsingular transformation $\widetilde{T}$ of $\widetilde{X}$ and a Borel map $\pi: \widetilde{X} \rightarrow X$ such that the following are satisfied:

- $\tilde{\mu} \circ \pi^{-1}=\mu$

- $\pi \widetilde{T}=T \pi$,

- $\omega_{\widetilde{T}, \widetilde{\mu}}$ is $\pi^{-1}(\mathfrak{B})$-measurable,

- $\bigvee_{n>0} \widetilde{T}^{n} \pi^{-1}(\mathfrak{B})=\widetilde{\mathfrak{B}}(\bmod \widetilde{\mu})$.

The dynamical system $(\tilde{X}, \widetilde{\mu}, \widetilde{T})$ is called the natural extentsion of $(X, \mu, T)$. The natural extension exists and it is unique up to a natural isomorphism (see [Si], [SiTh]). In the case when $T$ preserves a probability measure, the natural extension of $T$ coincides with the well known Rokhlin's natural extension of $T$.

Example 2.4 [DaHa]. Let $T$ be a one-sided shift on $X=\{0,1\}^{\mathbb{N}}$ endowed with an infinite product measure $\mu=\bigotimes_{i>0} \mu_{i}$, where $\mu_{i}$ is a distribution on $\{0,1\}$ such that $0<\mu_{i}(0)<1$ for each $i$. Since $\mu \circ T^{-1}=\bigotimes_{i>0} \mu_{i+1}$, it follows from the Kakutani theorem on equivalence of infinite product measures [Ka] that $T$ is an endomorphism of $(X, \mu)$, i.e. $T$ is $\mu$-nonsingular, if and only if

$$
\sum_{i=1}^{\infty}\left(\left(\sqrt{\mu_{i}(0)}-\sqrt{\mu_{i+1}(0)}\right)^{2}+\left(\sqrt{\mu_{i}(1)}-\sqrt{\mu_{i+1}(1)}\right)^{2}\right)<\infty .
$$

We let $\widetilde{X}:=\{0,1\}^{\mathbb{Z}}$ and $\widetilde{\mu}:=\bigotimes_{n \in \mathbb{Z}} \widetilde{\mu}_{n}$, where $\widetilde{\mu}_{n}=\mu_{1}$ if $n \leq 0$ and $\widetilde{\mu}_{n}=\mu_{n}$ if $n \geq 1$. Let $\widetilde{T}$ denote the two-sided shift on $\widetilde{X}$ and let $\pi: \widetilde{X} \rightarrow X$ denote the restriction map, i.e. $(\pi(x))_{n}=x_{n}$ for $n \geq 1$. Then

$$
\frac{d \widetilde{\mu} \circ \widetilde{T}}{d \widetilde{\mu}}(x)=\prod_{i \in \mathbb{Z}} \frac{\widetilde{\mu}_{i-1}\left(x_{i}\right)}{\widetilde{\mu}_{i}\left(x_{i}\right)}=\prod_{i \geq 2} \frac{\widetilde{\mu}_{i-1}\left(x_{i}\right)}{\widetilde{\mu}_{i}\left(x_{i}\right)}, \quad x \in \widetilde{X} .
$$

On the other hand, $\frac{d \mu}{d \mu \circ T^{-1}}(x)=\prod_{i \geq 1} \frac{\mu_{i}\left(x_{i}\right)}{\mu_{i+1}\left(x_{i}\right)}$. Thus $\frac{d \widetilde{\mu} \circ \widetilde{T}}{d \widetilde{\mu}}(x)=\frac{d \mu}{d \mu \circ T^{-1}}(T \pi(x))$ for a.a. $x \in X$. Hence $(\widetilde{X}, \widetilde{\mu}, \widetilde{T})$ is the natural extension of $(X, \mu, T)$.

A $\sigma$-finite measure $\nu$ on $(X, \mathfrak{B})$ is called $T$-cohomologous to $\mu$ if $\nu$ is equivalent to $\mu$ and the Radon-Nikodym derivative $d \nu / d \mu$ is measurable with respect to $T^{-1} \mathfrak{B}$.

\section{Lemma 2.5.}

(i) $(\widetilde{X}, \widetilde{\mu}, \widetilde{T})$ is conservative if and only if $T$ is $\mu$-recurrent [SiTh].

(ii) If $\nu$ is T-cohomologous to $\mu$ then the natural extensions $(\widetilde{X}, \widetilde{\mu}, \widetilde{T})$ and $(\widetilde{X}, \widetilde{\nu}, \widetilde{T})$ of $(X, \mu, T)$ and $(X, \nu, T)$ respectively are isomorphic [SiTh].

(iii) If $T$ is $\mu$-recurrent then $T$ is ergodic if and only if $\widetilde{T}$ is ergodic.

(iv) The Maharam extension $\widetilde{T}_{\omega_{\widetilde{T}, \widetilde{\mu}}}$ of $\widetilde{T}$ is canonically isomorphic to the natural extension $\widetilde{T_{\omega_{T, \mu}}}$ of the Maharam extension of $T$.

Let $R$ be an invertible nonsingular transformation on a $\sigma$-finite standard measure space $(X, \mathfrak{B}, \mu)$. Then $R$ is said to be a $K$-automorphism ([Pa], [SiTh]) if there is a $\sigma$-finite algebra $\mathfrak{F} \subset \mathfrak{B}$ such that

- $R^{-1} \mathfrak{F} \subset \mathfrak{F}$,

- the Radon-Nikodym derivative $\omega_{R, \mu}$ is $\mathfrak{F}$-measurable,

- $\bigvee_{n>0} R^{n} \mathfrak{F}=\mathfrak{B}(\bmod \mu)$,

- $\bigcap_{n>0} R^{-n} \mathfrak{F}=\{\emptyset, X\}(\bmod \mu)$. 
In other words, $R$ is a $K$-automorphisms if and only if it is the natural extension of an exact factor (semi-invariant sub- $\sigma$-algebra) of $R$.

Lemma 2.6 ([Pa], $[\mathrm{SiTh}])$. Each $K$-automorphism $R$ is either totally dissipative ${ }^{3}$ or conservative. In the latter case $R$ weakly mixing.

The following example generalizes $[\mathrm{Ha} \text {, Theorem } 1]^{4}$. Our proof is shorter and more elementary.

Example 2.7. Let $\tilde{X}=\{0,1\}^{\mathbb{Z}}$ and let $\mu=\bigotimes_{n \in \mathbb{Z}} \mu_{n}$ where $\mu_{n}(0):=p$ if $n \leq 0$ and $\mu_{n}(0):=q$ if $n>0$ for some positive reals $p, q \in(0,1)$. Let $\widetilde{T}$ denotes the two-sided shift on $\widetilde{X}$. Of course, if $p=q$ then $\widetilde{T}$ is conservative because $\widetilde{T}$ is a probability preserving Bernoulli shift. We now show that if $p \neq q$ then $\widetilde{T}$ is dissipative. Without loss of generality we may assume that $p<q$. For $\mu$-a.a. $x \in \widetilde{X}$, we have

$$
\frac{d \mu \circ \widetilde{T}}{d \mu}(x)=\prod_{n \in \mathbb{Z}} \frac{\mu_{n-1}\left(x_{n}\right)}{\mu_{n}\left(x_{n}\right)}=\frac{\mu_{0}\left(x_{1}\right)}{\mu_{1}\left(x_{1}\right)} .
$$

Therefore for each $n>0$,

$$
\frac{d \mu \circ \widetilde{T}^{n}}{d \mu}(x)=\frac{\mu_{0}\left(x_{1}\right) \cdots \mu_{0}\left(x_{n}\right)}{\mu_{1}\left(x_{1}\right) \cdots \mu_{1}\left(x_{n}\right)}=\left(\frac{p}{q}\right)^{n}\left(\frac{q(1-p)}{p(1-q)}\right)^{x_{1}+\cdots+x_{n}}
$$

at a.e. $x$. Since $p^{q}(1-p)^{1-q}<q^{q}(1-q)^{1-q}$, there is $\epsilon>0$ such that

$$
\delta:=\left(\frac{p}{q}\right)^{q-\epsilon}\left(\frac{1-p}{1-q}\right)^{1-q+\epsilon}<1 .
$$

It follows from the individual ergodic theorem (for the one-sided shift) that if $n$ is sufficiently large then $\frac{x_{1}+\cdots+x_{n}}{n} \leq 1-q+\epsilon$ for a.e. $x$. It follows from (2-2) and (2-3) that $\frac{d \mu \circ \widetilde{T}^{n}}{d \mu}(x) \leq \delta^{n}$. Therefore the series $\sum_{n \geq 1} \frac{d \mu \circ \widetilde{T}^{n}}{d \mu}(x)$ converges at a.e. $x$. Hence $\widetilde{T}$ is not $\mu$-recurrent. It follows from Lemma $2.3(i v)$ that $\widetilde{T}$ is dissipative. Moreover, in view of Example 2.4, $\widetilde{T}$ is a natural extension of an exact endomorphism. Hence, $\widetilde{T}$ is a $K$-automorphism. By Lemma $2.6, \widetilde{T}$ is totally dissipative.

\section{Nonsingular Bernoulli SHifts}

Throughout this section we will use the notation introduced in Example 2.4. Thus $(\widetilde{X}, \widetilde{\mu}, \widetilde{T})$ stands for the natural extension of the one-sided $\mu$-nonsingular Bernoulli shift $(X, \mu, T)$. In particular, $(2-1)$ holds. It is easy to verify that $\mathcal{S}_{T}$ is the tail equivalence relation on $X$. Hence $\mathcal{S}_{T}$ is ergodic by Kolmogorov's zeroone law. By Lemma 2.1(ii), $T$ is exact. Therefore $\widetilde{T}$ is a $K$-automorphism.

\section{Theorem 3.1.}

(i) If $\sum_{n=1}^{\infty}\left(\mu_{n}(0)-\mu_{1}(0)\right)^{2}<\infty$ then $\tilde{\mu}$ is equivalent to $\bigotimes_{n=-\infty}^{\infty} \mu_{1}{ }^{5}$.

(ii) If $\sum_{n=1}^{\infty}\left(\mu_{n}(0)-\mu_{1}(0)\right)^{2}=\infty$ and $\widetilde{T}$ is conservative then the Maharam extension $\widetilde{T}_{\omega_{\widetilde{T}, \widetilde{\mu}}}$ of $\widetilde{T}$ is a weakly mixing $K$-automorphism.

\footnotetext{
${ }^{3}$ We recall that an invertible transformation $R$ of a standard measure space $(Y, \nu)$ is called totally dissipative if there is a subset $Y_{0} \subset Y$ such that $R^{n} Y_{0} \cap Y_{0}=\emptyset$ for each $n \in \mathbb{N}$ and $\bigsqcup_{n \in \mathbb{Z}} R^{n} Y_{0}=Y$.

${ }^{4}$ Hamachi considers only the case $p=0.5$ in [Ha].

${ }^{5}$ Thus, in this case, $\widetilde{T}$ is isomorphic to the measure preserving Bernoulli shift on $\left(\widetilde{X}, \bigotimes_{n=-\infty}^{\infty} \mu_{1}\right)$.
} 
Proof. ( $i$ ) It follows from Lemma $1.3(i)$ that $\mu$ is equivalent to the infinite product $\nu:=\mu_{1} \times \mu_{1} \times \cdots$. Moreover, it is easy to see that the Radon-Nikodym derivative $d \mu / d \nu$ does not depend on $x_{1}$. Hence by Lemma $2.5(i i)$, the natural extension of $(T, \nu)$ is isomorphic to $\widetilde{T}$. On the other hand, according to Example 2.4, the natural extension of $(T, \nu)$ is the 2 -sided Bernoulli shift on $\left(\widetilde{X}, \bigotimes_{n \in \mathbb{Z}} \mu_{1}\right)$.

(ii) By the Maharam theorem, since $\widetilde{T}$ is conservative, $\widetilde{T}_{\omega_{\widetilde{T}, \widetilde{\mu}}}$ is also conservative. Hence in view of Lemma 2.6 , if $\widetilde{T}_{\omega_{\widetilde{T}, \tilde{\mu}}}$ is a $K$-automorphism then it is weakly mixing. Thus it suffices to prove that $\widetilde{T}_{\omega_{\widetilde{T}, \widetilde{\mu}}}$ is a $K$-automorphism. We will proceed in several steps. Let $\mathcal{L}$ stand for the set of limit points of the sequence $\left(\mu_{n}(0)\right)_{n \geq 1}$.

Claim A. $\mathcal{L} \ni \mu_{1}(0)$.

Indeed, if $\mathcal{L} \not \supset \mu_{1}(0)$ then there are $\delta>0$ and $N>0$ such that $\left|\mu_{1}(0)-\mu_{n}(0)\right|>\delta$ for each $n \geq N$. Therefore we have that

$$
\begin{aligned}
\int_{\widetilde{X}} \sqrt{\frac{d \widetilde{\mu} \circ \widetilde{T}^{n}}{d \widetilde{\mu}}(x) d \widetilde{\mu}(x)} & =\prod_{k \in \mathbb{Z}} \int \sqrt{\frac{d \mu_{k-n}}{d \mu_{k}}\left(x_{k}\right)} d \mu_{k}\left(x_{k}\right) \\
& =\prod_{k>0}\left(\sqrt{\mu_{k-n}(0) \mu_{k}(0)}+\sqrt{\mu_{k-n}(1) \mu_{k}(1)}\right) \\
& \leq \prod_{k=N}^{n}\left(\sqrt{\mu_{k-n}(0) \mu_{k}(0)}+\sqrt{\mu_{k-n}(1) \mu_{k}(1)}\right) \\
& =\prod_{k=N}^{n}\left(\sqrt{\mu_{1}(0) \mu_{k}(0)}+\sqrt{\mu_{1}(1) \mu_{k}(1)}\right) .
\end{aligned}
$$

Since $\eta:=\sup \left\{\sqrt{\mu_{1}(0) t}+\sqrt{\mu_{1}(1)(1-t)}|t \in(0,1)| t-,\mu_{1}(0) \mid>\delta\right\}<1$, we obtain that $\left\langle U_{\widetilde{T}}^{n} 1,1\right\rangle \leq \eta^{n-N}$. It now follows from Lemma 2.2 that $\widetilde{T}$ is dissipative. This contradicts to the condition of the theorem. Hence $\mu_{1}(0) \in \mathcal{L}$, as claimed.

Claim B. If $\mathcal{L}=\left\{\mu_{1}(0)\right\}$ then $\widetilde{T}_{\omega_{\widetilde{T}, \tilde{\mu}}}$ is a $K$-automorphism.

By Lemma 2.5 $(i v), \widetilde{T}_{\omega_{\widetilde{T}, \tilde{\mu}}}$ is isomorphic to the natural extension $\widetilde{T_{\omega_{T, \mu}}}$ of the endomorphism $T_{\omega_{T, \mu}}$. Hence $\widetilde{T}_{\omega_{\widetilde{T}, \widetilde{\mu}}}$ is a $K$-automorphism if $T_{\omega_{T, \mu}}$ is exact. By Lemma $2.1(i i), T_{\omega_{T, \mu}}$ is exact if and only if the equivalence relation $\mathcal{S}_{T_{\omega_{T, \mu}}}$ is ergodic. We note that $\mathcal{S}_{T_{\omega_{T, \mu}}}=\mathcal{S}_{T}\left(\alpha_{\omega_{T, \mu}} \uparrow \mathcal{S}_{T}\right)$. Thus it suffices to show that the cocycle $\alpha_{\omega_{T, \mu}}$ restricted to $\mathcal{S}_{T}$ is ergodic. Take $(x, y) \in S_{T}$. Then there is $n>0$ such that $T^{n} x=T^{n} y$, i.e. $x_{i}=y_{i}$ if $i>n$. Let $\widetilde{x}, \widetilde{y} \in \widetilde{X}$ be such that $\pi(\widetilde{x})=x$ and $\pi(\widetilde{y})=y$. Since

$\alpha_{\omega_{T, \mu}}\left(x, T^{n} x\right)=\omega_{T, \mu}(x) \cdots \omega_{T, \mu}\left(T^{n-1} x\right)=\omega_{\widetilde{T}, \widetilde{\mu}}(\widetilde{x}) \cdots \omega_{\widetilde{T}, \widetilde{\mu}}\left(\widetilde{T}^{n-1} \widetilde{x}\right)=\frac{d \widetilde{\mu} \circ \widetilde{T}^{n}}{d \widetilde{\mu}}(\widetilde{x})$

it follows that

$$
\alpha_{\omega_{T, \mu}}(x, y)=\frac{\alpha_{\omega_{T, \mu}}\left(x, T^{n} x\right)}{\alpha_{\omega_{T, \mu}}\left(y, T^{n} y\right)}=\frac{\frac{d \widetilde{\mu} \circ \widetilde{T}^{n}}{d \widetilde{\mu}}(\widetilde{x})}{\frac{d \widetilde{\mu} \circ \widetilde{T}^{n}}{d \widetilde{\mu}}(\widetilde{y})}
$$


This yields

$$
\begin{aligned}
\alpha_{\omega_{T, \mu}}(x, y) & =\frac{\prod_{i=1}^{n} \frac{\mu_{1}\left(x_{i}\right)}{\mu_{i}\left(x_{i}\right)} \prod_{i=n+1}^{\infty} \frac{\mu_{i-n}\left(x_{i}\right)}{\mu_{i}\left(x_{i}\right)}}{\prod_{i=1}^{n} \frac{\mu_{1}\left(y_{i}\right)}{\mu_{i}\left(y_{i}\right)} \prod_{i=n+1}^{\infty} \frac{\mu_{i-n}\left(y_{i}\right)}{\mu_{i}\left(y_{i}\right)}} \\
& =\prod_{i=1}^{n} \frac{\mu_{i}\left(y_{i}\right)}{\mu_{i}\left(x_{i}\right)} \prod_{i=1}^{n} \frac{\mu_{1}\left(x_{i}\right)}{\mu_{1}\left(y_{i}\right)} .
\end{aligned}
$$

Thus we obtain that $\alpha_{\omega_{T, \mu}}(x, y)=\Delta_{\mathcal{S}_{T}, \mu}(x, y) / \delta(x, y)$, where $\delta$ is a cocycle of $\mathcal{S}_{T}$ is given by $\delta(x, y)=\prod_{i=1}^{\infty} \mu_{1}\left(y_{i}\right) / \mu_{1}\left(x_{i}\right)$. It follows from Proposition 1.4 that $\alpha_{\omega_{T, \mu}}$ restricted to $\mathcal{S}_{T}$ is ergodic.

Claim $C$. If $\mu_{1}(0) \in \mathcal{L}$ but $\mathcal{L} \neq\left\{\mu_{1}(0)\right\}$ then $\widetilde{T}_{\omega_{\widetilde{T}, \tilde{\mu}}}$ is a $K$-automorphism.

As we have proved in Claim B, it suffices to show that $\alpha_{\omega_{T, \mu}} \uparrow \mathcal{S}_{T}$ is ergodic. Since $(2-1)$ is satisfied, there is a segment $[\alpha, \beta] \subset \mathcal{L}$ such that $\mu_{1}(0) \in[\alpha, \beta]$. Then it is easy to see that that we can find an infinite subset $I \subset \mathbb{N}$, a real $\lambda \in[\alpha, \beta]$ and a sequence $\epsilon_{i} \rightarrow 0$ such that the complement $\mathbb{N} \backslash I$ is infinite, $\mu_{i}(0)=\lambda e^{\epsilon_{i}}$ if $i \in I$ and $\sum_{i \in I} \epsilon_{i}^{2}=\infty$. Since $\mathcal{S}_{T}$ is naturally isomorphic to the direct product of the tail equivalence relations $\mathcal{S}^{1}$ and $\mathcal{S}^{2}$ on $\left(\{0,1\}^{I}, \bigotimes_{i \in I} \mu_{i}\right)$ and $\left(\{0,1\}^{\mathbb{N} \backslash I}, \bigotimes_{i \in \mathbb{N} \backslash I} \mu_{i}\right)$ respectively and the restriction of $\alpha_{\omega_{T, \mu}}$ to $\mathcal{S}^{1}$ is ergodic by Claim $\mathrm{B}$, it follows that $\alpha_{\omega_{T, \mu}} \uparrow \mathcal{S}_{T}$ is also ergodic.

\section{Remark 3.2.}

(i) We note that if the Bernoulli shift $(X, \mu, T)$ is equilibrial, i.e. $\mu_{1}(0)=\mu_{1}(1)$ then (and only in this case) $\alpha_{\omega_{T, \mu}} \uparrow \mathcal{S}_{T}=\Delta_{\mathcal{S}_{T}, \mu}$. Therefore in this case to prove Theorem 3.1 it would suffice to apply the well known Lemma 1.3(ii) instead of Propostion 1.4. That was done in [Ko2].

(ii) The Krengel entropy of $\widetilde{T}$ is infinite [SiTh].

The next statement follows immediately from Theorem 3.1.

Corollary 3.3. If $\sum_{n=1}^{\infty}\left(\mu_{1}(0)-\mu_{n}(0)\right)^{2}<\infty$ then $\widetilde{T}$ is of type II $I_{1}$. If $\sum_{n=1}^{\infty}\left(\mu_{1}(0)-\right.$ $\left.\mu_{n}(0)\right)^{2}=\infty$ and $\widetilde{T}$ is conservative then $\widetilde{T}$ is of type $I I I_{1}$.

\section{Forcing conservativeness of Bernoulli shifts}

Let $X=\{0,1\}^{\mathbb{Z}}$ and let

$$
\mathcal{A}_{n}:=\left\{\left[a_{n}, \ldots, a_{n}\right]_{-n}^{n} \mid a_{-n}, \ldots, a_{n} \in\{0,1\}\right\}
$$

Denote by $T$ the 2 -sided shift on $X$. We now state without proof a standard approximation result (cf. Lemma 1.1).

Lemma 4.1. Let $\mu$ be a probability measure on $X$ and let $T$ be $\mu$-nonsingular. If for each $n>0$ and $A \in \mathcal{A}_{n}$, there are a subset $A_{0} \subset A$ and a one-to-one transformation $\tau_{A}: A_{0} \rightarrow A$ such that $\mu\left(A_{0}\right)>0.9 \mu(A)$ and $\tau_{A} x \in\left\{T^{n} x \mid n>0\right\}$ and

$$
\left|\log \left(\frac{d \mu \circ \tau_{A}}{d \mu}(x)\right)\right|<0.001
$$

for a.e. $x \in A_{0}$ then $T$ is $\mu$-conservative. 
Fix $\lambda \in(0,1]$ and a sequence $\left(\epsilon_{n}\right)_{n \in \mathbb{Z}}$ of reals such that $\epsilon_{n}=0$ if $n \leq 1$ and

$$
\lim _{n \rightarrow \infty} \epsilon_{n}=0 .
$$

We define a measure $\mu$ on $X$ by setting

$$
\mu=\bigotimes_{n \in \mathbb{Z}} \mu_{n}, \text { where } \mu_{n}(0)=\frac{1}{1+\lambda e^{\epsilon_{n}}}, \mu_{n}(1)=\frac{\lambda e^{\epsilon_{n}}}{1+\lambda e^{\epsilon_{n}}} \text { for each } n \in \mathbb{Z} .
$$

It follows from Kakutani's theorem (see (2-1) and [Ka]), that $T$ is $\mu$-nonsingular if and only if

$$
\sum_{n=1}^{\infty}\left|\epsilon_{n+1}-\epsilon_{n}\right|^{2}<\infty
$$

According to Theorem 3.1, if $T$ is conservative and

$$
\sum_{n=1}^{\infty} \epsilon_{n}^{2}=\infty
$$

then $T$ is ergodic of type $I I I_{1}$. Thus our purpose is to construct $\left(\epsilon_{n}\right)_{n \geq 1}$ such that (4-1), (4-3) and (4-4) are satisfied and $T$ is conservative. We will do this inductively. Each step of the inductive construction will consist of two semi-steps. On the first semi-step we "do conservativeness" of $T$ partly, on $\mathcal{A}_{n}$. On the second semi-step we "satisfy partly" (4-1), (4-3) and (4-4). The only additional problem is that the second semi-step of the $m$-th step will affect the the property of partial conservativeness achieved on the $n$-th steps for $n<m$. Thus we have to control that the total contribution of the subsequent steps $(m>n)$ into the partial conservativeness of $T$ on $\mathcal{A}_{n}$ is "small".

Fix a sequence $\left(\eta_{n}\right)_{n=1}^{\infty}$ of positive reals such that $\eta_{n} \rightarrow 0$ as $n \rightarrow \infty$. Suppose that we have already defined $\epsilon_{1}, \ldots, \epsilon_{L_{n-1}}$. We now let

$$
\mu^{(n)}:=\bigotimes_{i \leq L_{n-1}} \mu_{i} \otimes \bigotimes_{i>L_{n-1}} \mu_{1}
$$

Since $\mu^{(n)}$ is equivalent to the infinite product $\bigotimes_{i \in \mathbb{Z}} \mu_{1}$, it follows that $T$ is $\mu^{(n)}$ nonsingular and $\mu^{(n)}$-conservative. Hence applying the standard exhaustion argument we can find for each cylinder $A \in \mathcal{A}_{L_{n-1}}$, positive integers $p_{1}, \ldots, p_{m}$ and pairwise disjoint cylinders $B_{1}, \ldots, B_{m} \subset A$ such that

$$
T^{p_{i}} B_{i} \subset A, T^{p_{i}} B_{i} \cap T^{p_{j}} B_{j}=\emptyset \text { if } 1 \leq i \neq j \leq m \text { and } \mu^{(n)}\left(\bigsqcup_{i=1}^{m} B_{i}\right)>0.9 \mu^{(n)}(A) \text {. }
$$

We now define a map $\tau_{A}: \bigsqcup_{i=1}^{m} B_{i} \rightarrow A$ by setting $\tau_{A} x:=T^{p_{i}} x$ if $x \in B_{i}$. Then $\tau_{A}$ is one-to-one and $\frac{d \mu^{(n)} \circ \tau_{A}}{d \mu^{(n)}}(x)=1$ for all $x \in \bigsqcup_{i=1}^{m} B_{i}$. Choose $\ell_{n}>L_{n-1}$ large so that $B_{i}$ and $T^{p_{i}} B_{i}$ are unions of cylinders from $\mathcal{A}_{\ell_{n}}$ for each $i$. It follows that $p_{i}<\ell_{n}$ for $i=1, \ldots, m$. We now set $\epsilon_{j}:=0$ if $L_{n-1}<j \leq \ell_{n}$.

Now we choose an integer $L_{n}>2 \ell_{n}$ and reals $\epsilon_{\ell_{n}+1} \geq \cdots \geq \epsilon_{L_{n}}$ so that

$$
\sum_{j=\ell_{n}+1}^{2 \ell_{n}} \epsilon_{j}<\eta_{n}, \sum_{j=\ell_{n}+1}^{L_{n}}\left(\epsilon_{j-1}-\epsilon_{j}\right)^{2}<\eta_{n} \text { and } \sum_{j=\ell_{n}+1}^{L_{n}} \epsilon_{j}^{2}>1 .
$$

Thus we defined $\epsilon_{1}, \ldots, \epsilon_{L_{n}}$. Continuing this construction process infinitely many times, we obtain an increasing sequence $\ell_{1}<L_{1}<\ell_{2}<L_{2}<\cdots$ and a sequence $\left(\epsilon_{n}\right)_{n \geq 1}$. Moreover, for each cylinder $A \in \bigcup_{n \geq 1} \mathcal{A}_{L_{n}}$, we have a map $\tau_{A}$ satisfying the properties listed above. 
Theorem 4.2. Let $\lambda \in(0,1)$ and let $\left(\epsilon_{n}\right)_{n>0}$ be a sequence of nonnegative reals defined via the aforementioned inductive procedure. Define $\mu$ by (4-2) utilizing $\lambda$ and $\left(\epsilon_{n}\right)_{n>0}$. Then the 2-sided shift $T$ on $(X, \mu)$ is $\mu$-nonsingular, $\mu$-conservative and of type $I I I_{1}$.

Proof. Since (4-5) implies (4-1), (4-3) and (4-4), $T$ is $\mu$-nonsingular and of type $I I I_{1}$ whenever it is $\mu$-conservative (in view of Corollary 3.3). Thus it suffices to verify that $T$ is $\mu$-conservative. For that we will apply Lemma 4.1.

We first note that $\mu(C)=\mu^{(n)}(C)$ for each cylinder $C \in \mathcal{A}_{\ell_{n}}, n>0$. Take a cylinder $A \in \mathcal{A}_{L_{n-1}}$. By the definition of $\mu$, there are mutually disjoint cylinders $B_{1}, \ldots, B_{m} \in \mathcal{A}_{\ell_{n}}$, positive integers $p_{1}, \ldots, p_{m}<\ell_{n}$ and a one-to-one map $\tau_{A}: \bigsqcup_{i=1}^{m} B_{i} \rightarrow A$ such that $\mu^{(n)}\left(\bigsqcup_{i=1}^{m} B_{i}\right)>0.9 \mu^{(n)}(A), \tau_{A} x=T^{p_{i}} x$ and $\frac{d \mu^{(n)} \circ T^{p_{i}}}{d \mu^{(n)}}(x)=1$ for each $x \in B_{i}$. It follows that $\mu\left(\bigsqcup_{i=1}^{m} B_{i}\right)>0.9 \mu(A)$ and

$$
\frac{d \mu \circ T^{p_{i}}}{d \mu}(x)=\frac{d \mu}{d \mu^{(n)}}\left(T^{p_{i}} x\right) \frac{d \mu^{(n)}}{d \mu}(x) \quad \text { for each } x \in B_{i} .
$$

Since $\frac{d \mu}{d \mu^{(n)}}(x)=\prod_{k>n} \prod_{j=\ell_{k}+1}^{L_{k}} \frac{\mu_{j}\left(x_{j}\right)}{\mu_{1}\left(x_{j}\right)}$ for each $x \in X$, we obtain that

$$
\frac{d \mu \circ T^{p_{i}}}{d \mu}(x)=\prod_{k>n} \prod_{j=\ell_{k}+1}^{L_{k}} \frac{\mu_{j}\left(x_{j+p_{i}}\right) \mu_{1}\left(x_{j}\right)}{\mu_{j}\left(x_{j}\right) \mu_{1}\left(x_{j+p_{i}}\right)}=\prod_{k>n} \prod_{j=\ell_{k}+1}^{L_{k}+p_{i}} \frac{\mu_{j-p_{i}}\left(x_{j}\right)}{\mu_{j}\left(x_{j}\right)}
$$

for each $x \in B_{i}$. Given $0<a<b$ and $p>0$, we have

$$
\prod_{j=a}^{b} \frac{\mu_{j-p}\left(x_{j}\right)}{\mu_{j}\left(x_{j}\right)}=\prod_{j=a}^{b} \frac{\left(\lambda e^{\epsilon_{j-p}}\right)^{x_{j}}}{1+\lambda e^{\epsilon_{j-p}}} \cdot \frac{1+\lambda e^{\epsilon_{j}}}{\left(\lambda e^{\epsilon_{j}}\right)^{x_{j}}}=\prod_{j=a}^{b} e^{x_{j}\left(\epsilon_{j-p}-\epsilon_{j}\right)} \frac{1+\lambda e^{\epsilon_{j}}}{1+\lambda e^{\epsilon_{j-p}}} .
$$

We note that $\epsilon_{j-p} \geq \epsilon_{j}$ if $L_{n}+p \geq j \geq \ell_{n}+1+p$ and $p<\ell_{n}$. Hence

$$
-\sum_{j=\ell_{n}+1}^{\ell_{n}+p} \epsilon_{j} \leq \sum_{j=\ell_{n}+1}^{L_{n}+p} x_{j}\left(\epsilon_{j-p}-\epsilon_{j}\right) \leq \sum_{j=\ell_{n}+1+p}^{L_{n}+p}\left(\epsilon_{j-p}-\epsilon_{j}\right)=\sum_{j=\ell_{n}+1}^{\ell_{n}+p} \epsilon_{j} .
$$

On the other hand, we see that

$$
\prod_{j=\ell_{n}+1}^{L_{n}+p} \frac{1+\lambda e^{\epsilon_{j}}}{1+\lambda e^{\epsilon_{j-p}}}=\frac{\prod_{j=L_{n}+1}^{L_{n}+p}\left(1+\lambda e^{\epsilon_{j}}\right)}{\prod_{j=\ell_{n}+1-p}^{\ell_{n}}\left(1+\lambda e^{\epsilon_{j}}\right)}=\frac{(1+\lambda)^{p}}{(1+\lambda)^{p}}=1 .
$$

This, (4-6)-(4-8) and (4-5) yield that for each $x \in \bigsqcup_{i=1}^{m} B_{i}$,

$$
\log \left|\frac{d \mu \circ \tau_{A}}{d \mu}(x)\right|=\log \left|\frac{d \mu \circ T^{p_{i}}}{d \mu}(x)\right| \leq \sum_{j=\ell_{n}+1}^{\ell_{n}+p_{i}} \epsilon_{j} \leq \eta_{n}
$$

where $i$ is chosen so that $x \in B_{i}$. Hence $T$ is $\mu$-conservative by Lemma 4.1. 


\section{MARKOV MEASURES}

Let $X=\{0,1\}^{\mathbb{N}}$. Given a distribution $\lambda$ on $\{0,1\}$ and a sequence $\boldsymbol{P}:=\left(P^{(n)}\right)_{n=1}^{\infty}$ of stochastic $2 \times 2$ matrices $P^{(n)}=\left(P_{i, j}^{(n)}\right)_{i, j=0,1}$, we define a Borel measure $\mu$ on $X$ by setting

$$
\mu\left(\left[a_{1}, \ldots, a_{k}\right]_{1}^{k}\right):=\lambda\left(a_{1}\right) P_{a_{1}, a_{2}}^{(1)} P_{a_{2}, a_{3}}^{(2)} \cdots P_{a_{k-1}, a_{k}}^{(k-1)} .
$$

It is called the Markov measure determined by the pair $(\lambda, \boldsymbol{P})$. We say that $\mu$ is non-degenerate if $\lambda(a)>0$ and $P_{a, b}^{(n)}>0$ for all $a, b \in\{0,1\}$ and $n>0$. It is easy to see that $\mu$ is non-atomic if and only if $\prod_{n=1}^{\infty} P_{x_{n}, x_{n+1}}^{(n)}=0$ for each $x \in X$. Let $\mathcal{R}$ denote the tail equivalence relation on $X$. If $\mu$ is non-degenerate then $\mathcal{R}$ is $\mu$-nonsingular. Indeed, it is straightforward to verify that

$$
\Delta_{\mathcal{R}, \mu}(x, y)=\frac{\lambda\left(y_{1}\right)}{\lambda\left(x_{1}\right)} \prod_{j=1}^{\infty} \frac{P_{y_{j}, y_{j+1}}^{(j)}}{P_{x_{j}, x_{j+1}}^{(j)}}
$$

where the product is, in fact, finite because $x$ and $y$ are $\mathcal{R}$-equivalent. The following theorem is a generalization of the well known Kakutani theorem on equivalence of infinite product measures [Ka].

Lemma 5.1 [Lo]. Let $\mu$ and $\nu$ be two Markov measures on $X$ determined by pairs $(\lambda, \boldsymbol{P})$ and $(\kappa, \boldsymbol{Q})$ respectively. Let the $\sigma$-algebra of $\mathcal{R}$-invariant subsets be trivial $(\bmod \nu)$ and let $\mu$ be non-degenerate. Then $\nu$ is absolutely continuous with respect to $\mu$ if and only if

$$
\int_{X} \sqrt{\frac{\kappa\left(x_{1}\right) Q_{x_{1}, x_{2}}^{(1)} Q_{x_{2}, x_{3}}^{(2)} \cdots Q_{x_{k-1}, x_{k}}^{(k-1)}}{\lambda\left(x_{1}\right) P_{x_{1}, x_{2}}^{(1)} P_{x_{2}, x_{3}}^{(2)} \cdots P_{x_{k-1}, x_{k}}^{(k-1)}}} d \mu(x) \not \nrightarrow 0
$$

as $k \rightarrow \infty$.

A natural question arises: under which conditions on $\mu$ the $\sigma$-algebra of Borel $\mathcal{R}$-invariant subsets is trivial $(\bmod \mu)$ ? To answer it, we state a theorem from [BrDo] which is an analog of Kolmogorov zero-one law for the general probability measures on $X$. For that we need a piece of notation. Denote by $\mathfrak{B}_{n}$ the (finite) $\sigma$-algebra generated by cylinders $\left[a_{1}, \ldots, a_{n}\right]_{1}^{n}, a_{1}, \ldots, a_{n} \in\{0,1\}$. Denote by $\mathfrak{B}^{n}$ the smallest $\sigma$-algebra such that the maps $X \ni x \mapsto x_{k} \in\{0,1\}, k>n$, are all measurable. Let $\mu$ be a probability measure on $X$. For each $n>0$, we denote by $\widehat{\mu}_{n}$ the following probability measure on $X$ determined by

$$
\widehat{\mu}_{n}(A \cap B)=\mu(A) \mu(B), \quad A \in \mathfrak{B}_{n}, B \in \mathfrak{B}^{n} .
$$

This measure is equivalent to $\mu$. We let $r_{n}:=d \widehat{\mu}_{n} / d \mu$.

Lemma 5.2 [BrDo]. Let $\mu$ be a probability measure on $X$. The $\sigma$-algebra of Borel $\mathcal{R}$-invariant subsets is trivial $(\bmod \mu)$ if and only if $E\left(r_{n} \mid \mathfrak{B}_{n} \vee \mathfrak{B}^{l}\right) \rightarrow 1$ in measure $\mu$ as $l \rightarrow \infty$ for each $n \geq 1$.

Let $\mu$ be a Markov measure determined by $(\lambda, P)$. Given $m>n>0$, we denote by $P^{(n, m)}$ the matrix product $P^{(n)} P^{(n+1)} \cdots P^{(m)}$. Of course, $P^{(n, m)}$ is also a stochastic matrix. 
Lemma 5.3. If $\mu$ is non-degenerate and non-atomic then for each $n>0$ and $a, b, c \in\{0,1\}$, there exists a limit

$$
\lim _{m \rightarrow \infty} \frac{P_{a, c}^{(n, m)}}{P_{b, c}^{(n, m)}}=1 .
$$

Proof. Of course, it suffices to verify only the case $a=c=0$ and $b=1$. To this end, we first note that for each stochastic $2 \times 2$ matrix $A=\left(\begin{array}{cc}a_{0,0} & a_{0,1} \\ a_{1,0} & a_{1,1}\end{array}\right)$ with non-zero entries, $|\operatorname{det} A|=\left|a_{0,0}-a_{1,0}\right|<1$. Hence either $\prod_{m=n}^{\infty}\left|\operatorname{det} P^{(m)}\right|=0$ for each $n>0$ or $\prod_{m=n}^{\infty}\left|\operatorname{det} P^{(m)}\right|>0$ for some $n>0$. In the latter case, we obtain that

$$
\prod_{m=n}^{\infty} \max \left(P_{0,0}^{(m)}, P_{1,0}^{(m)}\right) \geq \prod_{m=n}^{\infty}\left|P_{0,0}^{(m)}-P_{1,0}^{(m)}\right|=\prod_{m=n}^{\infty}\left|\operatorname{det} P^{(m)}\right|>0 .
$$

This contradicts to the assumption that $\mu$ is non-atomic. Hence

$$
\lim _{m \rightarrow \infty}\left|P_{0,0}^{(n, m)}-P_{1,0}^{(n, m)}\right|=\lim _{m \rightarrow \infty}\left|\operatorname{det} P^{(n, m)}\right|=\prod_{m=n}^{\infty}\left|\operatorname{det} P^{(m)}\right|=0
$$

for each $n>0$. Moreover, the sequence $\left(\min \left(P_{0,0}^{(n, m)}, P_{1,0}^{(n, m)}\right)\right)_{m>n}$ increases and the sequence $\left(\max \left(P_{0,0}^{n, m}, P_{1,0}^{n, m}\right)\right)_{m>n}$ decreases as $m \rightarrow \infty$ for each $n$. Hence $(5-2)$ implies that $\frac{P_{0,0}^{(n, m)}}{P_{1,0}^{(n, m)}} \rightarrow 1$ as $m \rightarrow \infty$.

Theorem 5.4. Let $\mu$ be a non-degenerate and non-atomic Markov measure determined by $(\lambda, \boldsymbol{P})$. Then $\mathcal{R}$ is $\mu$-ergodic.

Proof. Let $\mu=\int \mu_{x} d \nu(x)$ stand for the disintegration of $\mu$ with respect to the restriction $\nu$ of $\mu$ to $\mathfrak{B}_{n} \vee \mathfrak{B}^{l-1}$. For each finite sequence $a_{1}, \ldots, a_{m} \in\{0,1\}$ with $m>l$, we have $\mu_{x}\left(\left[a_{1}, \ldots, a_{m}\right]_{1}^{m}\right)=0$ if $a_{j} \neq x_{j}$ for some $j \in\{1, \ldots, n\} \cup\{l, \ldots m\}$ and

$$
\begin{aligned}
\mu_{x}\left(\left[a_{1}, \ldots, a_{m}\right]_{1}^{m}\right) & =\frac{\mu\left(\left[x_{1}, \ldots, x_{n}, a_{n+1}, \ldots a_{l-1}, x_{l}, \ldots, x_{m}\right]_{l}^{m}\right)}{\mu\left(\left[x_{1}, \ldots, x_{n}\right]_{1}^{n} \cap\left[x_{l}, \ldots, x_{m}\right]_{l}^{m}\right)} \\
& =\frac{P_{x_{n}, a_{n+1}}^{(n)} P_{a_{n+1}, a_{n+2}}^{(n+1)} \cdots P_{a_{l-1}, x_{l}}^{(l-1)}}{\sum_{b_{n+1}, \ldots, b_{l-1}=0}^{1} P_{x_{n}, b_{n+1}}^{(n)} P_{b_{n+1}, b_{n+2}}^{(n+1)} \cdots P_{b_{l-1}, x_{l}}^{(l-1)}} \cdot \frac{\mu\left(\left[x_{n}\right]_{n}\right)}{\mu\left(\left[x_{n}\right]_{n}\right)} \\
& =\frac{\mu\left(\left[x_{n}, a_{n+1}, \ldots, a_{l-1}, x_{l}\right]_{n}^{l}\right)}{\mu\left(\left[x_{n}\right]_{n} \cap\left[x_{l}\right]_{l}\right)}
\end{aligned}
$$

otherwise. We also have that

$$
\begin{aligned}
\frac{d \widehat{\mu}_{n}}{d \mu}(x) & =\lim _{m \rightarrow \infty} \frac{\widehat{\mu}_{n}\left(\left[x_{1}, \ldots, x_{m}\right]_{1}^{m}\right)}{\mu\left(\left[x_{1}, \ldots, x_{m}\right]_{1}^{m}\right)} \\
& =\lim _{m \rightarrow \infty} \frac{\mu\left(\left[x_{1}, \ldots, x_{n}\right]_{1}^{n}\right) \mu\left(\left[x_{n+1}, \ldots, x_{m}\right]_{n+1}^{m}\right)}{\mu\left(\left[x_{1}, \ldots, x_{m}\right]_{1}^{m}\right)} \\
& =\lim _{m \rightarrow \infty} \frac{\mu\left(\left[x_{n}\right]_{n}\right) \mu\left(\left[x_{n+1}\right]_{n+1}\right)}{\mu\left(\left[x_{n}, x_{n+1}\right]_{n}^{n+1}\right)} \\
& =\frac{\mu\left(\left[x_{n+1}\right]_{n+1}\right)}{P_{x_{n}, x_{n+1}}^{(n)}} .
\end{aligned}
$$


This yields

$$
\begin{aligned}
E\left(r_{n} \mid \mathfrak{B}_{n} \vee \mathfrak{B}^{l-1}\right)(x) & =\int \frac{\mu\left(\left[t_{n+1}\right]_{n+1}\right)}{P_{t_{n}, t_{n+1}}^{(n)}} d \mu_{x}(t) \\
& =\sum_{i=0}^{1} \mu\left([i]_{n+1}\right) \frac{\mu\left(\left[x_{n}, i\right]_{n}^{n+1} \cap\left[x_{l}\right]_{l}\right)}{P_{x_{n}, i}^{(n)} \mu\left(\left[x_{n}\right]_{n} \cap\left[x_{l}\right]_{l}\right)} \\
& =\sum_{i=0}^{1} \mu\left([i]_{n+1}\right) \frac{P_{i, x_{l}}^{(n+1, l-1)}}{\sum_{j=0}^{1} P_{x_{n}, j}^{(n)} P_{j, x_{l}}^{(n+1, l-1)}} .
\end{aligned}
$$

It follows from Lemma 5.3 and the condition of the theorem that $E\left(r_{n} \mid \mathfrak{B}_{n} \vee\right.$ $\left.\mathfrak{B}^{l-1}\right)(x) \rightarrow 1$ at a.e. $x \in X$. By Lemma $5.2, \mathcal{R}$ is $\mu$-ergodic.

Corollary 5.5. Let $\mu$ and $\nu$ be two non-degenerate and non-atomic Markov measures on $X$ determined by pairs $(\lambda, \boldsymbol{P})$ and $(\kappa, \boldsymbol{Q})$ respectively. Then $\nu$ is equivalent to $\mu$ if and only if

$$
\lim _{n \rightarrow \infty} \sum_{a_{1}=0}^{1} \sqrt{\kappa\left(a_{1}\right) \lambda\left(a_{1}\right)} \prod_{i=1}^{n-1} \sum_{a_{i+1}=0}^{1} \sqrt{Q_{a_{i}, a_{i+1}}^{(i)} P_{a_{i}, a_{i+1}}^{(i)}} \neq 0 .
$$

Moreover, in this case,

$$
\frac{d \nu}{d \mu}(x)=\frac{\kappa\left(x_{1}\right)}{\lambda\left(x_{1}\right)} \prod_{j=1}^{\infty} \frac{Q_{x_{j}, x_{j+1}}^{(j)}}{P_{x_{j}, x_{j+1}}^{(j)}}
$$

If $\inf _{n>0} \min _{i, j} P_{i, j}^{(n)}>0$ and $\inf _{n>0} \min _{i, j} Q_{i, j}^{(n)}>0$ then $\mu$ and $\nu$ are equivalent if and only if $\sum_{n=1}^{\infty}\left(P_{i, j}^{(n)}-Q_{i, j}^{(n)}\right)^{2}<\infty$ for each $i, j \in\{0,1\}$.

Proof. The first claim follows directly from Lemma 5.1 and Theorem 5.4 because the integral in (5-1) equals

$$
\begin{aligned}
\sum_{a_{1}, \ldots, a_{n}=0}^{1} \sqrt{\kappa\left(a_{1}\right) \lambda\left(a_{1}\right)} \prod_{i=1}^{n-1} \sqrt{Q_{a_{i}, a_{i+1}}^{(i)} P_{a_{i}, a_{i+1}}^{(i)}} & \\
& =\sum_{a_{1}=0}^{1} \sqrt{\kappa\left(a_{1}\right) \lambda\left(a_{1}\right)} \prod_{i=1}^{n-1} \sum_{a_{i+1}=0}^{1} \sqrt{Q_{a_{i}, a_{i+1}}^{(i)} P_{a_{i}, a_{i+1}}^{(i)}} .
\end{aligned}
$$

The second claim follows from the fact that $\mu \sim \nu$ and

$$
\frac{d \nu}{d \mu}(x)=\lim _{n \rightarrow \infty} \frac{\nu\left(\left[x_{1}, \ldots, x_{n}\right]_{1}^{n}\right)}{\mu\left(\left[x_{1}, \ldots, x_{n}\right]_{1}^{n}\right)}
$$

for a.e. $x \in X$. The final claim was proved in [Lo].

We can rewrite (5-3) formally in the following form

$$
\sum_{a_{1}=0}^{1} \sqrt{\kappa\left(a_{1}\right) \lambda\left(a_{1}\right)} \prod_{i=1}^{\infty} \sum_{a_{i+1}=0}^{1} \sqrt{Q_{a_{i}, a_{i+1}}^{(i)} P_{a_{i}, a_{i+1}}^{(i)}} \neq 0,
$$

which is close to the classical Kakutani criterium from [Ka]. 


\section{KRIEGER'S TYPE OF TAIL EQUIVALENCE RELATIONS EQUIPPED WITH STATIONARY MARKOV MEASURES}

In this section we compute Krieger's type of the tail equivalence relation $\mathcal{R}$ on $X$ equipped with "stationary" Markov measures.

Proposition 6.1. Let $\lambda$ be a non-degenerate distribution on $\{0,1\}$. Given a nondegenerate stochastic matrix $P$, we let $\boldsymbol{P}:=\left(P^{(n)}\right)_{n=1}^{\infty}$ with $P^{(n)}=P$ for each $n>0$. Denote by $\mu$ the Markov measure on $X$ determined by $(\lambda, \boldsymbol{P})$. Denote by $\Gamma$ the subgroup of $\mathbb{R}_{+}^{*}$ generated by reals $\frac{P_{0,0}}{P_{1,1}}$ and $\frac{P_{0,0}^{2}}{P_{0,1} P_{1,0}}$.

- If $\Gamma=\{1\}$ then $\mathcal{R}$ on $(X, \mu)$ is ergodic and of type $I I_{1}$.

- If $\Gamma=\left\{\lambda^{n} \mid n \in \mathbb{Z}\right\}$ for some $\lambda \in(0,1)$ then $\mathcal{R}$ on $(X, \mu)$ is ergodic and of type $I I I_{\lambda}$.

- If $\Gamma$ is dense in $\mathbb{R}_{+}^{*}$ then $\mathcal{R}$ on $(X, \mu)$ is ergodic and of type $I I I_{1}$.

Proof. We first note that $\mathcal{R}$ is ergodic by Theorem 5.4. The restriction of $\mathcal{R}$ to the subset $[0]_{1}$ is isomorphic to $\mathcal{R}$. Hence Krieger's type of $\mathcal{R} \cap\left([0]_{1} \times[0]_{1}\right)$ equals Krieger's type of $\mathcal{R}$. Given $k>0$, we define a transformation $\delta_{k}$ of $X$ by setting for each $x=\left(x_{j}\right)_{j>0}$ and $j>0$,

$$
\left(\delta_{k} x\right)_{j}= \begin{cases}x_{j} & \text { if } j \neq k \\ x_{k}^{*} & \text { if } j=k,\end{cases}
$$

where $x_{k}^{*}$ is determined by the condition $\left\{x_{k}, x_{k}^{*}\right\}=\{0,1\}$. Then $\delta_{k}$ is an invertible $\mu$-nonsingular transformation of $X$. Denote by $\Lambda$ the group of transformations of $X$ generated by $\delta_{k}, k=2,3, \ldots$. Then two points $x, y \in[0]_{1}$ are $\mathcal{R}$-equivalent if and only if $y \in\{\delta x \mid \delta \in \Lambda\}$. Hence $\Delta_{\mathcal{R}, \mu}(x, y) \in\left\{\frac{d \mu \circ \delta}{d \mu}(x) \mid \delta \in \Lambda\right\}$. It is straightforward to verify that

$$
\frac{d \mu \circ \delta_{k}}{d \mu}(x) \in\left\{\frac{P_{0,0}}{P_{1,1}}, \frac{P_{1,1}}{P_{0,0}}, \frac{P_{0,0}^{2}}{P_{0,1} P_{1,0}}, \frac{P_{0,1} P_{1,0}}{P_{0,0}^{2}}, \frac{P_{1,0} P_{0,1}}{P_{1,1}^{2}}, \frac{P_{1,1}^{2}}{P_{1,0} P_{0,1}}\right\}
$$

for each $x \in X$ and $k>1$. It follows from this and the cocycle identity that the Radon-Nikodym cocycle $\Delta_{\mathcal{R}, \mu}$ restricted to $\mathcal{R} \cap\left([0]_{1} \times[0]_{1}\right)$ takes its values in $\Gamma$. Therefore it suffices to show that $\frac{P_{1,1}}{P_{0,0}}$ and $\frac{P_{0,1} P_{1,0}}{P_{0,0}^{2}}$ are essential values of this cocycle. Take a cylinder $\left[0, a_{2}, \ldots, a_{n}\right]_{1}^{n} \subset[0]_{1}$. Let $N>n$ and let $A:=\left[0, a_{2}, \ldots, a_{n}\right]_{1}^{n} \cap[0,0,0]_{N-1}^{N+1}$ and $B:=\left[0, a_{2}, \ldots, a_{n}\right]_{1}^{n} \cap[0,0,1]_{N-1}^{N+1}$. By the Perron-Frobenius theorem, there is a strictly positive vector $\left(\pi_{0}, \pi_{1}\right)$ such that $\lim _{k \rightarrow \infty}\left(P^{k}\right)_{i, j}=\pi_{j}$ for each $i, j=0,1$. Given $\epsilon>0$, if $N$ is large enough then

$$
\begin{aligned}
& \frac{\mu(A)}{\mu\left(\left[0, a_{2}, \ldots, a_{n}\right]_{1}^{n}\right)}=\left(P^{N-n}\right)_{a_{n}, 0} P_{0,0}^{2} \geq(1-\epsilon) \pi_{0} P_{0,0}^{2} \quad \text { and } \\
& \frac{\mu(B)}{\mu\left(\left[0, a_{2}, \ldots, a_{n}\right]_{1}^{n}\right)}=\left(P^{N-n}\right)_{a_{n}, 0} P_{0,0} P_{0,1} \geq(1-\epsilon) \pi_{0} P_{0,0} P_{0,1} .
\end{aligned}
$$

Moreover, $\delta_{N} A \subset\left[0, a_{2}, \ldots, a_{n}\right]_{1}^{n}, \delta_{N} B \subset\left[0, a_{2}, \ldots, a_{n}\right]_{1}^{n}$,

$$
\begin{aligned}
& \frac{d \mu \circ \delta_{N}}{d \mu}(x)=\frac{P_{0,1} P_{1,0}}{P_{0,0}^{2}} \quad \text { for all } x \in A \text { and } \\
& \frac{d \mu \circ \delta_{N}}{d \mu}(x)=\frac{P_{0,1} P_{1,1}}{P_{0,0} P_{0,1}}=\frac{P_{1,1}}{P_{0,0}} \quad \text { for all } x \in B .
\end{aligned}
$$

Now Lemma 1.1 yields that $\frac{P_{0,1} P_{1,0}}{P_{0,0}^{2}}$ and $\frac{P_{1,1}}{P_{0,0}}$ are essential values of $\Delta_{\mathcal{R}, \mu} \uparrow[0]_{1}$. 
Corollary 6.2. Let $\alpha, \beta \in(0, \infty)$ and let $P=\left(\begin{array}{cc}\frac{1}{1+\alpha} & \frac{\alpha}{1+\alpha} \\ \frac{\beta}{1+\beta} & \frac{1}{1+\beta}\end{array}\right)$. Then $\mathcal{R}$ is of type:

(i) $I I_{1}$ if and only if $\alpha=\beta=1$;

(ii) $I I I_{\lambda}$ for some $\lambda \in(0,1)$ if and only if there are integers $p, q \in \mathbb{Z}$ such that $p \mathbb{Z}+q \mathbb{Z}=\mathbb{Z}, \alpha$ is the positive root of the quadratic equation

$$
\begin{gathered}
\alpha^{2}+\left(1-\lambda^{p}\right) \alpha-\lambda^{q}=0 \quad \text { and } \\
\beta=\lambda^{q-p} / \alpha ;
\end{gathered}
$$

(iii) $I I I_{1}$ if $\mathcal{R}$ is neither of type $I I_{1}$ nor of type $I I I_{\lambda}$ for $\lambda \in(0,1)$.

Proof. In view of Proposition 6.1, the claim (i) is obvious.

(ii) It follows from Proposition 6.1 that $\mathcal{R}$ is of type $I I I_{\lambda}$ if and only if there are relatively prime integers $p, q \in \mathbb{Z}$ such that

$$
\frac{1+\beta}{1+\alpha}=\lambda^{-p} \quad \text { and } \quad \frac{1+\beta}{(1+\alpha) \alpha \beta}=\lambda^{-q}
$$

This implies (6-1). We also note that the pair $(\alpha, \beta)=(1,1)$ can not be the solution of (6-1) because this would imply that $p=q=0$ and hence $p \mathbb{Z}+q \mathbb{Z} \neq \mathbb{Z}$.

(iii) follows from (i), (ii) and Proposition 6.1.

\section{Nonsingular Markov Shifts}

Let $\mu$ be a non-degenerate and non-atomic Markov measure determined by some pair $(\lambda, \boldsymbol{P})$. Let $T$ denote the one-sided shift on $(X, \mu)$. It is straightforward to verify that $\mu \circ T^{-1}$ is also a Markov measure on $X$. This measure is determined by a pair $(\widehat{\lambda}, \widehat{\boldsymbol{P}})$, where $\widehat{\lambda}(a)=\sum_{i=0}^{1} \lambda(i) P_{i, a}^{(1)}$ for $a=0,1$, and $\widehat{\boldsymbol{P}}=\left(\widehat{P}^{(n)}\right)_{n=1}^{\infty}$ with $\widehat{P}^{(n)}=P^{(n+1)}$ for $n>0$. It follows from Corollary 5.5 that $T$ is $\mu$-nonsingular if and only if

$$
\sum_{a_{1}=0}^{1} \sqrt{\widehat{\lambda}\left(a_{1}\right) \lambda\left(a_{1}\right)} \prod_{i=1}^{\infty} \sum_{a_{i+1}=0}^{1} \sqrt{P_{a_{i}, a_{i+1}}^{(i+1)} P_{a_{i}, a_{i+1}}^{(i)}} \neq 0
$$

In this case, for $\mu$-a.a. $x \in X$, we have

$$
\frac{d \mu \circ T^{-1}}{d \mu}(x)=\frac{\lambda(0) P_{0, x_{1}}^{(1)}+\lambda(1) P_{1, x_{1}}^{(1)}}{\lambda\left(x_{1}\right)} \prod_{k=1}^{\infty} \frac{P_{x_{k}, x_{k+1}}^{(k+1)}}{P_{x_{k}, x_{k+1}}^{(k)}} .
$$

Definition 7.1. We call the dynamical system $(X, \mu, T)$ the nonsingular one-sided Markov shift if $\mu$ is a non-degenerate and non-atomic Markov measure determined by $(\lambda, \boldsymbol{P})$ such that (7-1) holds.

Since $\mathcal{S}_{T}=\mathcal{R}$, it follows from Theorem 5.4 and Lemma 2.1(ii) that all nonsingular one-sided Markov shift are exact (and hence ergodic). We now describe the natural extensions of these nonsingular endomorphisms. 
Example 7.2. Let $(X, \mu, T)$ be a nonsingular one-sided Markov shift as above. Denote by $\widetilde{X}, \widetilde{T}$ and $\pi$ the same objects as in Example 2.4. Then $\widetilde{T}$ is the twosided shift on $\widetilde{X}$. To define the corresponding measure $\widetilde{\mu}$ on $\widetilde{X}$, we first set

$$
Q_{i, j}:=\frac{\lambda(i) P_{i, j}^{(1)}}{\lambda(0) P_{0, j}^{(1)}+\lambda(1) P_{1, j}^{(1)}}, \quad i, j=0,1 .
$$

Then $Q:=\left(\begin{array}{ll}Q_{0,0} & Q_{0,1} \\ Q_{1,0} & Q_{1,1}\end{array}\right)$ is a left stochastic matrix, i.e. $\sum_{i=0}^{1} Q_{i, j}=1$ for each $j \in\{0,1\}$. We now let

$$
\widetilde{\mu}\left(\left[a_{-n}, \ldots, a_{0}, \ldots, a_{n}\right]_{-n}^{n}\right)=\left(\prod_{-n<i \leq 1} Q_{a_{i-1}, a_{i}}\right) \lambda\left(a_{1}\right) \prod_{1 \leq i<n} P_{a_{i}, a_{i+1}}^{(i)} .
$$

It is straightforward to verify that $\widetilde{T}$ is $\widetilde{\mu}$-nonsingular and for $\widetilde{\mu}$-a.e. $\widetilde{x}=\left(\widetilde{x}_{k}\right)_{k \in \mathbb{Z}} \in$ $\widetilde{X}$, we have

$$
\frac{d \widetilde{\mu} \circ \widetilde{T}}{d \widetilde{\mu}}(\widetilde{x})=\frac{Q_{x_{1}, x_{2}} \lambda\left(x_{2}\right)}{\lambda\left(x_{1}\right) P_{x_{1}, x_{2}}^{(1)}} \cdot \prod_{k=2}^{\infty} \frac{P_{x_{k}, x_{k+1}}^{(k-1)}}{P_{x_{k}, x_{k+1}}^{(k)}} .
$$

We use here the notation $x:=\pi(\widetilde{x}), x=\left(x_{n}\right)_{n>0}$ and hence $x_{n}=\widetilde{x}_{n}$ for each $n>0$. On the other hand, (7-2) yields that

$$
\frac{d \mu}{d \mu \circ T^{-1}}(T x)=\frac{\lambda\left(x_{2}\right)}{\lambda(0) P_{0, x_{2}}^{(1)}+\lambda(1) P_{1, x_{2}}^{(1)}} \cdot \prod_{k=2}^{\infty} \frac{P_{x_{k}, x_{k+1}}^{(k-1)}}{P_{x_{k}, x_{k+1}}^{(k)}} .
$$

This, (7-3) and (7-4) yield that $\frac{d \widetilde{\mu} \circ \widetilde{T}}{d \widetilde{\mu}}(\widetilde{x})=\frac{d \mu}{d \mu \circ T^{-1}} \circ T(\pi(\widetilde{x}))$ for $\widetilde{\mu}$-a.e. $\widetilde{x}$, i.e. $\omega_{\widetilde{T}, \widetilde{\mu}}=\omega_{T, \mu} \circ \pi$. Let $\mathfrak{B}$ and $\widetilde{\mathfrak{B}}$ denote the standard Borel $\sigma$-algebras on $X$ and $\widetilde{X}$ respectively. Since $\bigvee_{n \in \mathbb{Z}} \widetilde{T}^{n} \pi^{-1}(\mathfrak{B})=\widetilde{\mathfrak{B}}$, it follows that $\widetilde{T}$ is the natural extension of $T$, as desired. Since $T$ is exact, $\widetilde{T}$ is a $K$-automorphism. We also deduce from (7-4) that for each $n>0$,

$$
\frac{d \widetilde{\mu} \circ \widetilde{T}^{n}}{d \widetilde{\mu}}(\widetilde{x})=\frac{\lambda\left(x_{n+1}\right)}{\lambda\left(x_{1}\right)} \prod_{i=1}^{n} \frac{Q_{x_{i}, x_{i+1}}}{P_{x_{i}, x_{i+1}}^{(i)}} \prod_{i=n+1}^{\infty} \frac{P_{x_{i}, x_{i+1}}^{(i-n)}}{P_{x_{i}, x_{i+1}}^{(i)}}
$$

at $\widetilde{\mu}$-a.e. $\widetilde{x}$. We will utilize this formula below.

Lemma 7.3. Let $(X, \mu, T)$ be a nonsingular one-sided Markov shift and let the measure $\mu$ be determined by a pair $(\lambda, \boldsymbol{P})$. Then $\alpha_{\omega_{T, \mu}} \uparrow \mathcal{S}_{T}=\Delta_{\mathcal{S}_{T}, \mu} / \delta$, where $\delta: \mathcal{S}_{T} \rightarrow \mathbb{R}_{+}^{*}$ is a cocycle of $\mathcal{S}_{T}$ given by $\delta(x, y)=\prod_{i=1}^{\infty} Q_{y_{i}, y_{i+1}} / Q_{x_{i}, x_{i+1}}$.

Proof. As in the proof of Theorem 3.1 (see Claim B) take $(x, y) \in S_{T}$. Then there is $n>0$ such that $T^{n} x=T^{n} y$, i.e. $x_{i}=y_{i}$ if $i>n$. Let $\widetilde{x}, \widetilde{y} \in \widetilde{X}$ be such that $\pi(\widetilde{x})=x$ and $\pi(\widetilde{y})=y$. We have that

$$
\alpha_{\omega_{T, \mu}}(x, y)=\frac{\alpha_{\omega_{T, \mu}}\left(x, T^{n} x\right)}{\alpha_{\omega_{T, \mu}}\left(y, T^{n} y\right)}=\frac{\frac{d \widetilde{\mu} \circ \widetilde{T}^{n}}{d \widetilde{\mu}}(\widetilde{x})}{\frac{d \widetilde{\mu} \circ \widetilde{T}^{n}}{d \widetilde{\mu}}(\widetilde{y})} .
$$


Applying (7-5) we obtain that

$$
\begin{aligned}
\alpha_{\omega_{T, \mu}}(x, y) & =\frac{\frac{\lambda\left(x_{n+1}\right)}{\lambda\left(x_{1}\right)} \prod_{i=1}^{n} \frac{Q_{x_{i}, x_{i+1}}}{P_{x_{i}, x_{i+1}}^{(i)}} \prod_{i=n+1}^{\infty} \frac{P_{x_{i}, x_{i+1}}^{(i-n)}}{P_{x_{i}, x_{i+1}}^{(i)}}}{\frac{\lambda\left(y_{n+1}\right)}{\lambda\left(y_{1}\right)} \prod_{i=1}^{n} \frac{Q_{y_{i}, y_{i+1}}}{P_{y_{i}, y_{i+1}}^{(i)}} \prod_{i=n+1}^{\infty} \frac{P_{y_{i}, y_{i+1}}^{(i-n)}}{P_{y_{i}, y_{i+1}}^{(i)}}} \\
& =\frac{\lambda\left(y_{1}\right)}{\lambda\left(x_{1}\right)} \prod_{i=1}^{n} \frac{P_{y_{i}, y_{i+1}}^{(i)}}{P_{x_{i}, x_{i+1}}^{(i)}} \prod_{i=1}^{n} \frac{Q_{x_{i}, x_{i+1}}}{Q_{y_{i}, y_{i+1}}}
\end{aligned}
$$

Hence $\alpha_{\omega_{T, \mu}}(x, y)=\Delta_{\mathcal{S}_{T}, \mu}(x, y) / \delta(x, y)$, as desired.

Remark 7.4. The following assertions are verified straightforwardly.

(i) $\delta$ is trivial if and only if $Q_{i, j}=0.5$ for all $i, j=0,1$. This happens if only if $\lambda(0)=\lambda(1)=0.5$ and $P_{0, j}^{(1)}=P_{1, j}^{(1)}$ for $j=0,1$.

(ii) If $P^{(1)}$ is bistochastic then $Q$ is bistochastic if and only if $\lambda(0)=\lambda(1)=0.5$. In this case we have $Q=P^{(1)}$ and

$$
\delta(x, y)=\left(\frac{P_{0,1}^{(1)}}{P_{0,0}^{(1)}}\right)^{\sum_{i=1}^{\infty}\left(\left|y_{i+1}-y_{i}\right|-\left|x_{i+1}-x_{i}\right|\right)}
$$

for all $(x, y) \in \mathcal{S}_{T}$.

(iii) $Q_{i, 0}=Q_{i, 1}$ if and only if $P_{0, i}^{(1)}=P_{1, i}^{(1)}, i=0,1$.

Theorem 7.5. Let $(X, \mu, T)$ be a nonsingular one-sided Markov shift. If the cocycle $\Delta_{\mathcal{S}_{T}, \mu} / \delta$ is ergodic then the Maharam extension of the natural extension $(\tilde{X}, \widetilde{\mu}, \widetilde{T})$ of $T$ is a K-automorphism. If, moreover, $\widetilde{T}$ is conservative then $\widetilde{T}$ is weakly mixing and of type $I I I_{1}$.

Idea of the proof. Repeat the argument in the beginning of Claim B from the proof of Theorem 3.1 almost literally and then apply Lemma 7.3.

We now prove a necessary condition for conservativeness of $\widetilde{T}$.

Lemma 7.6. Let $P_{0,0}^{(1)}=P_{1,0}^{(1)}$. If there exist $\eta>0$ and $k>0$ such that $\mid Q_{b, a}-$ $P_{a, b}^{(n)} \mid>\eta$ for all $a, b \in\{0,1\}$ and $n>k$ then $\widetilde{T}$ is not conservative.

Proof. By the condition of the lemma and Remark 7.4(iii), there is a probability distribution $q$ on $\{0,1\}$ such that $Q_{a, b}=q(a)$ for all $a, b \in\{0,1\}$.

It follows from (7-5) and Fatou's lemma that

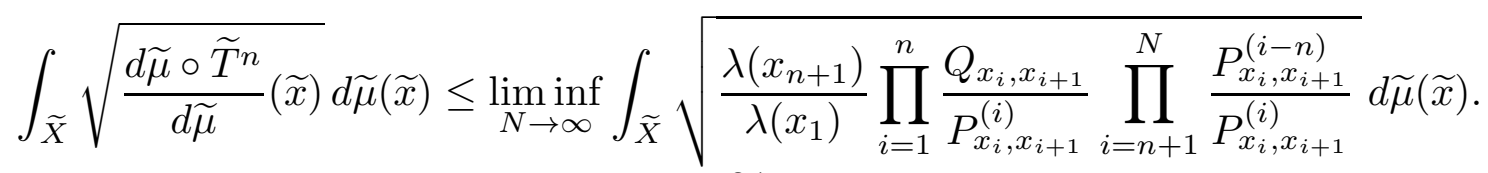


The integral in the righthand side of this inequality equals

$$
\begin{aligned}
& \sum_{a_{1}, \ldots, a_{N+1}=0}^{1} \sqrt{\frac{\lambda\left(a_{n+1}\right)}{\lambda\left(a_{1}\right)} \prod_{i=1}^{n} \frac{Q_{a_{i}, a_{i+1}}}{P_{a_{i}, a_{i+1}}^{(i)}} \prod_{i=n+1}^{N} \frac{P_{a_{i}, a_{i+1}}^{(i-n)}}{P_{a_{i}, a_{i+1}}^{(i)}}} \cdot \mu\left(\left[a_{1} \ldots a_{N+1}\right]_{1}^{N+1}\right) \\
& =\sum_{a_{1}, \ldots, a_{N+1}=0}^{1} \sqrt{\lambda\left(a_{n+1}\right) \lambda\left(a_{1}\right) \prod_{i=1}^{n} Q_{a_{i}, a_{i+1}} P_{a_{i}, a_{i+1}}^{(i)} \prod_{i=n+1}^{N} P_{a_{i}, a_{i+1}}^{(i-n)} P_{a_{i}, a_{i+1}}^{(i)}} \\
& =\sum_{a_{1}, \ldots, a_{n+1}=0}^{1} \sqrt{\lambda\left(a_{n+1}\right) \lambda\left(a_{1}\right) \prod_{i=1}^{n} Q_{a_{i}, a_{i+1}} P_{a_{i}, a_{i+1}}^{(i)}} \prod_{i=n+1}^{N} \sum_{a_{i+1}=0}^{1} \sqrt{P_{a_{i}, a_{i+1}}^{(i-n)} P_{a_{i}, a_{i+1}}^{(i)}} .
\end{aligned}
$$

Since $\sum_{s=0}^{1} \sqrt{P_{u, s}^{(i-n)} P_{u, s}^{(i)}} \leq 1$ and $\lambda(u)<1$ for each $u=0,1$ and $i=n+1, \ldots, N$, it follows that

$$
\begin{aligned}
\int_{\widetilde{X}} \sqrt{\frac{d \widetilde{\mu} \circ \widetilde{T}^{n}}{d \widetilde{\mu}}(\widetilde{x})} d \widetilde{\mu}(\widetilde{x}) \leq \sum_{a_{1}, \ldots, a_{n+1}=0}^{1} \sqrt{\prod_{i=1}^{n} Q_{a_{i}, a_{i+1}} P_{a_{i}, a_{i+1}}^{(i)}} \\
=\sum_{a_{1}, \ldots, a_{n+1}=0}^{1} \sqrt{q\left(a_{1}\right)} \sqrt{\prod_{i=1}^{n-1} P_{a_{i}, a_{i+1}}^{(i)} q\left(a_{i+1}\right)} \sqrt{P_{a_{n}, a_{n+1}}^{(n)}} \\
\leq 2 \sum_{a_{1}, \ldots, a_{n}=0}^{1} \sqrt{\prod_{i=2}^{n} P_{a_{i-1}, a_{i}}^{(i-1)} q\left(a_{i}\right)} \\
=2 \sum_{a_{1}, \ldots, a_{k+1}=0}^{1} \sqrt{\prod_{i=2}^{k+1} P_{a_{i-1}, a_{i}}^{(i-1)} q\left(a_{i}\right)} \prod_{i=k+2}^{n} \sum_{a_{i}=0}^{1} \sqrt{P_{a_{i-1}, a_{i}}^{(i-1)} q\left(a_{i}\right)} .
\end{aligned}
$$

Since there is $\xi<1$ such that

$$
\sup \{\sqrt{t q(a)}+\sqrt{(1-t)(1-q(a))}|t \in(0,1),| t-q(a) \mid \geq \eta\} \leq \xi,
$$

it follows from the condition of the proposition that

$$
\prod_{i=k+2}^{n} \sum_{a_{i}=0}^{1} \sqrt{P_{a_{i-1}, a_{i}}^{(i-1)} q\left(a_{i}\right)} \leq \xi^{n-k-1} .
$$

This yields

$$
\int_{\widetilde{X}} \sqrt{\frac{d \widetilde{\mu} \circ \widetilde{T}^{n}}{d \widetilde{\mu}}(\widetilde{x})} d \widetilde{\mu}(\widetilde{x}) \leq 2 \xi^{n-k-1} \prod_{i=k+2}^{n} \sum_{a_{i}=0}^{1} \sqrt{P_{a_{i-1}, a_{i}}^{(i-1)} q\left(a_{i}\right)} \leq 2 \xi^{n-k-1} .
$$

Therefore $\sum_{n=1}^{\infty}\left\langle U_{\widetilde{T}}^{n} 1,1\right\rangle<\infty$. Hence $\widetilde{T}$ is not conservative by Lemma 2.2 . 


\section{Maharam extensions of Markov shifts. Bistochastic Case}

Let $\mu$ be a (non-degenerate and non-atomic) Markov measure determined by a pair $(\lambda, \boldsymbol{P})$ for a sequence $\boldsymbol{P}=\left(P^{(n)}\right)_{n=1}^{\infty}$ of bistochastic $2 \times 2$ matrices $P^{(n)}, n \geq 1$. It is convenient now to identify $\{0,1\}$ with the group $\mathbb{Z} / 2 \mathbb{Z}$. Then the space $X$ can be considered as the compact Abelian group $(\mathbb{Z} / 2 \mathbb{Z})^{\mathbb{N}}$. Let $\theta: X \rightarrow X$ denote the following group homomorphism

$$
X \ni x=\left(x_{1}, x_{2}, x_{3}, \ldots\right) \mapsto\left(x_{1}, x_{2}-x_{1}, x_{3}-x_{2}, \ldots\right) \in X .
$$

This homomorphism has been proved to be useful in [Do-Qu]. Of course, $\theta$ is one-to-one and continuous. For each $y=\left(y_{1}, y_{2}, \ldots\right) \in X$, we have that $\theta\left(y_{1}, y_{1}+\right.$ $\left.y_{2}, y_{1}+y_{2}+y_{3}, \ldots\right)=y$. Hence $\theta$ is onto. Thus $\theta$ is a (topological) automorphism of $X$. Since $P^{(n)}$ is bistochastic, it follows that $P_{a+c, b+c}^{(n)}=P_{a, b}^{(n)}$ for all $a, b, c \in \mathbb{Z} / 2 \mathbb{Z}$ and $n>0$. This yields

$$
\begin{aligned}
\mu \circ \theta^{-1}\left(\left[y_{1}, \ldots, y_{n}\right]_{1}^{n}\right) & =\mu\left(\left[y_{1}, y_{1}+y_{2}, \ldots, y_{1}+\cdots+y_{n}\right]_{1}^{n}\right) \\
& =\lambda\left(y_{1}\right) P_{y_{1}, y_{1}+y_{2}}^{(1)} P_{y_{1}+y_{2}, y_{1}+y_{2}+y_{3}}^{(2)} \cdots P_{y_{1}+\cdots+y_{n-1}, y_{1}+\cdots+y_{n}}^{(n-1)} \\
& =\lambda\left(y_{1}\right) P_{0, y_{2}}^{(1)} P_{0, y_{3}}^{(2)} \cdots P_{0, y_{n}}^{(n-1)} .
\end{aligned}
$$

Hence $\mu \circ \theta^{-1}$ is a Bernoulli measure $\bigotimes_{n=1}^{\infty} \mu_{n}$ on $X$, where $\mu_{1}:=\lambda$ and $\mu_{n}(i):=$ $P_{0, i}^{(n-1)}, i=0,1$ and $n>1$. We claim that

$$
(\theta \times \theta)(\mathcal{R})=\mathcal{R}_{0}:=\left\{(x, y) \in \mathcal{R} \mid \sum_{i=1}^{\infty}\left(y_{i}-x_{i}\right)=0\right\} .
$$

The inclusion $(\theta \times \theta)(\mathcal{R}) \subset \mathcal{R}_{0}$ is obvious. Conversely, if $(x, y) \in \mathcal{R}_{0}$, then there is $N>0$ such that $y_{1}+\cdots+y_{N}=x_{1}+\cdots+x_{N}$ and $x_{i}=y_{i}$ for each $i>N$. Then $\left(\theta^{-1} x, \theta^{-1} y\right)=\left(\left(x_{1}, x_{1}+x_{2}, \ldots\right),\left(y_{1}, y_{1}+y_{2}, \ldots\right)\right) \in \mathcal{R}$. Thus (8-1) is proved.

Let $T$ stand for the one-sided shift on $X$. It is easy to verify that

$$
\theta T \theta^{-1}\left(y_{1}, y_{2}, y_{3}, \ldots\right)=\left(y_{1}+y_{2}, y_{3}, y_{4}, \ldots\right)
$$

for each $\left(y_{1}, y_{2}, \ldots\right) \in X$. Of course, $T$ is $\mu$-nonsingular if and only if $\theta T \theta^{-1}$ is $\mu \circ \theta^{-1}$-nonsingular. In turn, the latter holds if and only if $T$ is $\mu \circ \theta^{-1}$-nonsingular. Indeed, for $a \in \mathbb{Z} / 2 \mathbb{Z}$, we set $A_{a}:=\left\{(u, v) \in(\mathbb{Z} / 2 \mathbb{Z})^{2} \mid u+v=a\right\}$. Then we have

$$
\begin{aligned}
\frac{d \mu \circ T^{-1}}{d \mu}\left(\theta^{-1} y\right) & =\frac{d\left(\mu \circ \theta^{-1}\right) \circ \theta T^{-1} \theta^{-1}}{d \mu \circ \theta^{-1}}(y) \\
& =\frac{\left(\mu_{1} \otimes \mu_{2}\right)\left(A_{y_{1}}\right)}{\mu_{1}\left(y_{1}\right)} \prod_{n \geq 2} \frac{\mu_{n+1}\left(y_{n}\right)}{\mu_{n}\left(y_{n}\right)} \\
& =\frac{\left(\mu_{1} \otimes \mu_{2}\right)\left(A_{y_{1}}\right)}{\mu_{2}\left(y_{1}\right)} \cdot \frac{d\left(\mu \circ \theta^{-1}\right) \circ T^{-1}}{d\left(\mu \circ \theta^{-1}\right)}(y)
\end{aligned}
$$

for $\mu \circ \theta^{-1}$-a.e. $y=\left(y_{1}, y_{2}, \ldots\right) \in X$. Therefore $T$ is $\mu$-nonsingular if and only if $T$ is $\mu \circ \theta^{-1}$-nonsingular. Equivalently, in the bistochastic case, (7-1) is equivalent to $(2-1)$. We then call the dynamical system $(X, \mu, T)$ a bistochastic nonsingular one-sided Markov shift. Let $(\widetilde{X}, \widetilde{\mu}, \widetilde{T})$ denote the natural extension of $(X, \mu, T)$. 
Theorem 8.1. Let $(X, \mu, T)$ be a bistochastic nonsingular one-sided Markov shift as above with $\lambda(0)=\lambda(1)=0.5$ and $P^{(1)}=\left(\begin{array}{ll}0.5 & 0.5 \\ 0.5 & 0.5\end{array}\right)$. Then the following are satisfied.

(i) $(\widetilde{X}, \widetilde{\mu}, \widetilde{T})$ is conservative if and only if $\left(\widetilde{X}, \widetilde{\mu \circ \theta^{-1}}, \widetilde{T}\right)$ is conservative.

(ii) The Maharam extension of $(\widetilde{X}, \widetilde{\mu}, \widetilde{T})$ is a $K$-automorphism if the cocycle $\Delta_{\mathcal{R}_{0}, \mu \circ \theta^{-1}}$ is ergodic.

(iii) If $(\widetilde{X}, \widetilde{\mu}, \widetilde{T})$ is conservative then it is weakly mixing and either of type II $I_{1}$ (if $\left.\sum_{n \geq 1}\left(P_{0,0}^{(n)}-0.5\right)^{2}<\infty\right)$ or of type III $I_{1}$ (otherwise). In the latter case, the Maharam extension of $(\widetilde{X}, \widetilde{\mu}, \widetilde{T})$ is a K-automorphism.

Proof. It follows from the assumption of the theorem that $\left(\mu_{1} \otimes \mu_{2}\right)\left(A_{a}\right)=\mu_{1}(a)$ for each $a \in \mathbb{Z} / 2 \mathbb{Z}$ and $\mu_{2}=\mu_{1}$. Then (8-2) yields that $\omega_{T, \mu \circ \theta^{-1}}=\omega_{T, \mu} \circ \theta^{-1}$. Lemma 2.3(vi) yields now that $T$ is $\mu$-recurrent if and only if $T$ is $\mu \circ \theta^{-1}$-recurrent. Hence, $(i)$ follows from Lemma 2.5(i).

It follows from Remark 7.4(i) and the assumption of the theorem that the cocycle $\delta$ is trivial. Then, by Theorem 7.5, the Maharam extension of $\widetilde{T}$ is a $K$ automorphism if the cocycle $\Delta_{\mathcal{S}_{T}, \mu}$ is ergodic. It remains to note that (see (8-1))

$$
\Delta_{\mathcal{S}_{T}, \mu}\left(\theta^{-1} x, \theta^{-1} y\right)=\Delta_{\theta \times \theta\left(\mathcal{S}_{T}\right), \mu \circ \theta^{-1}}(x, y)=\Delta_{\mathcal{R}_{0}, \mu \circ \theta^{-1}}(x, y)
$$

for all $(x, y) \in \mathcal{S}_{T}$. Thus $(i i)$ is proved.

Since $\mathcal{R}_{0}$ contains the equivalence relation generated by the group of finite permutations of $\mathbb{N}$ acting on $X$ in the natural way, it follows from Remark 1.7 that $\Delta_{\mathcal{R}_{0}, \mu \circ \theta^{-1}}$ is ergodic if and only if $\Delta_{\mathcal{R}, \mu \circ \theta^{-1}}$ is ergodic. Therefore arguing as in the proof of Theorem 3.1 (see also Corollary 3.3) and utilizing (i) and (ii) we prove $(i i i)$.

We note that, under the condition of the theorem, if $(\widetilde{X}, \widetilde{\mu}, \widetilde{T})$ is conservative and of type $I I_{1}$ then $\mu$ is $T$-cohomologous to a Markov measure determined by the pair $\left(\lambda,\left(P^{(1)}\right)_{n=1}^{\infty}\right)$. Thus $\mu$ is $T$-cohomologous to a Bernoulli (i.e. infinite product) measure. The converse follows from the proposition below:

Proposition 8.2. Let $(X, \mu, T)$ be as in Theorem 8.1. If $\mu$ is equivalent to a Bernoulli measure then $\widetilde{T}$ is ergodic and of type $I I_{1}$.

Proof. Let $\mu$ be equivalent to a Markov measure determined by a pair $\left(\rho,\left(V^{(n)}\right)_{n=1}^{\infty}\right)$ with $V_{0,0}^{(n)}=V_{1,0}^{(n)}$ for all $n>0$. Then $V^{(n)}$ is close to $P^{(n)}$ for all sufficiently large $n$ by Corollary 5.5. Since $P^{(n)}$ is bistochastic, it follows that $V_{0,0}^{(n)} \rightarrow 0.5$ as $n \rightarrow \infty$. If follows that there is $\delta>0$ such that $\inf _{n>0} \min _{i, j} V_{i, j}^{(n)}>\delta$. Hence there is $\delta^{\prime}>0$ such that $\inf _{n>0} \min _{i, j} P_{i, j}^{(n)}>\delta^{\prime}$. It follows from Corollary 5.5 that $\sum_{n=1}^{\infty}\left(P_{0,0}^{(n)}-V_{0,0}^{(n)}\right)^{2}<\infty$ and $\sum_{n=1}^{\infty}\left(1-P_{0,0}^{(n)}-V_{0,0}^{(n)}\right)^{2}<\infty$. Therefore $\sum_{n=1}^{\infty}\left(P_{0,0}^{(n)}-0.5\right)^{2}<\infty$ and hence $\sum_{n=1}^{\infty}\left(P_{i, j}^{(n)}-0.5\right)^{2}<\infty$ for all $i, j \in\{0,1\}$. It remains to apply Corollary 5.5 again (but in the opposite direction).

Remark 8.3. Utilizing Theorem 8.1 we can produce concrete examples of type $I I I_{1}$ ergodic conservative natural extensions of bistochastic nonsingular one-sided Markov shifts. For that take a concrete example of a nonsingular one-sided Bernoulli 
shift $\left(X, \bigotimes_{n=1}^{\infty} \mu_{n}, T\right)$ whose natural extension is conservative ergodic and of type $I I I_{1}$ such that $\mu_{1}(0)=\mu_{2}(0)=0.5$ (such systems were constructed in Theorem 4.2 and earlier in $[\mathrm{Kr}],[\mathrm{Ha}],[\mathrm{Ko} 1]$ and $[\mathrm{Ko} 2])$ and set $\mu:=\left(\bigotimes_{n=1}^{\infty} \mu_{n}\right) \circ \theta$. Then the system $(\widetilde{X}, \widetilde{\mu}, \widetilde{T})$ is as desired. We note also that $\mu$ is not equivalent to any Bernoulli measure on $X$ according to Proposition 8.2.

\section{Maharam extensions of Markov shifts. General Case}

Let $J$ be an infinite subset of $\mathbb{N}$ such that its complement $\mathbb{N} \backslash J$ is also infinite. We endow $J$ and $\mathbb{N} \backslash J$ with the induced (from $\mathbb{N}$ ) linear ordering. Using this ordering we may identify naturally $\{0,1\}^{J}$ with $X$ and $\{0,1\}^{\mathbb{N} \backslash J}$ with $X$. We denote by $\phi_{J}: X \ni x \mapsto x \mid J \in X$ and $\phi_{\mathbb{N} \backslash J}: X \ni x \mapsto x \mid(\mathbb{N} \backslash J) \in X$ the corresponding restriction maps. Then the mapping

$$
X \ni x \mapsto\left(\phi_{J} \times \phi_{\mathbb{N} \backslash J}\right)(x) \in X \times X
$$

is a homeomorphism. Moreover, the mapping $\left(\phi_{J} \times \phi_{\mathbb{N} \backslash J}\right) \times\left(\phi_{J} \times \phi_{\mathbb{N} \backslash J}\right)$ maps bijectively $\mathcal{R}$ onto $\mathcal{R} \times \mathcal{R}$.

Let $\mu$ be a probability measure on $X$ such that $\mathcal{R}$ is $\mu$-nonsingular. In view of the identification (9-1), we may consider $\mu$ as a measure on $X \times X$. Then $\mathcal{R} \times \mathcal{R}$ is $\mu$-nonsingular. Now we let $\mu_{J}:=\mu \circ \phi_{J}^{-1}$. Then $\mu$ admits a disintegration

$$
\mu=\int_{X} \delta_{x} \times \mu^{(x)} d \mu_{J}(x)
$$

relative to $\mu_{J}$, where $\delta_{x}$ is the Kronecker measure supported at $x$ and $X \ni x \mapsto \mu^{(x)}$ is the corresponding canonical system of conditional measures on $X$. Since $\mathcal{R} \times \mathcal{R}$ is $\mu$-nonsingular, $\mathcal{R}$ is $\mu^{(x)}$-nonsingular for $\mu_{J}$-a.a. $x \in X$. Moreover, $\mu^{(x)}$ and $\mu^{\left(x^{\prime}\right)}$ are equivalent whenever $\left(x, x^{\prime}\right) \in \mathcal{R}$ and

$$
\Delta_{\mathcal{R} \times \mathcal{R}, \mu}\left((x, y),\left(x^{\prime}, y^{\prime}\right)\right)=\Delta_{\mathcal{R}, \mu_{J}}\left(x, x^{\prime}\right) \frac{d \mu^{\left(x^{\prime}\right)}}{d \mu^{(x)}}(y) \Delta_{\mathcal{R}, \mu^{\left(x^{\prime}\right)}}\left(y, y^{\prime}\right) .
$$

Lemma 9.1. Suppose that $\mathcal{R} \times \mathcal{R}$ is $\mu$-ergodic. If $\left(\mathcal{R}, \mu^{(x)}\right)$ is ergodic and of type $I I I_{1}$ for $\mu_{J}$-a.e. $x \in X$ then $(\mathcal{R} \times \mathcal{R}, \mu)$ is also of type $I I I_{1}$.

Proof. Denote the skew product equivalence relation $(\mathcal{R} \times \mathcal{R})\left(\Delta_{\mathcal{R} \times \mathcal{R}, \mu}\right)$ on the product space $\left(X \times X \times \mathbb{R}_{+}^{*}, \mu \times \lambda_{\mathbb{R}_{+}^{*}}\right)$ by $\widetilde{\mathcal{R}}$. We need to show that $\widetilde{\mathcal{R}}$ is ergodic. Let $A$ be an $\widetilde{\mathcal{R}}$-invariant subset of $X \times X \times \mathbb{R}_{+}^{*}$. Given $x \in X$, we let

$$
A_{x}:=\left\{(y, z) \in X \times \mathbb{R}_{+}^{*} \mid(x, y, z) \in A\right\} .
$$

Since $A$ is $\widetilde{\mathcal{R}}$-invariant, it follows from (9-3) that $A_{x}$ is $\mathcal{R}\left(\Delta_{\mathcal{R}, \mu_{x}}\right)$-invariant for $\mu_{J}$-a.e. $x \in X$. Indeed, $(x, y) \sim\left(x, y^{\prime}\right)$ whenever $\left(y, y^{\prime}\right) \in \mathcal{R}$ and hence (9-3) yields

$$
\Delta_{\mathcal{R} \times \mathcal{R}, \mu}\left((x, y),\left(x, y^{\prime}\right)\right)=\Delta_{\mathcal{R}, \mu^{(x)}}\left(y, y^{\prime}\right) .
$$

By the assumptions of the lemma, $\left(\mathcal{R}, \mu^{(x)}\right)$ is ergodic and of type $I I I_{1}$. Hence the skew product extension $\mathcal{R}\left(\Delta_{\mathcal{R}, \mu^{(x)}}\right)$ is ergodic. Therefore we have either $\left(\mu_{x} \times\right.$ $\left.\lambda_{\mathbb{R}_{+}^{*}}\right)\left(A_{x}\right)=0$ or $\left(\mu_{x} \times \lambda_{\mathbb{R}_{+}^{*}}\right)\left(\left(X \times \mathbb{R}_{+}^{*}\right) \backslash A_{x}\right)=0$ for a.e. $x \in X$. We let

$$
\begin{aligned}
& B:=\left\{x \in X \mid\left(\mu_{x} \times \lambda_{\mathbb{R}_{+}^{*}}\right)\left(\left(X \times \mathbb{R}_{+}^{*}\right) \backslash A_{x}\right)=0\right\} \quad \text { and } \\
& \widetilde{B}:=\left\{(x, y, z) \in X \times X \times \mathbb{R}_{+}^{*} \mid x \in B\right\} .
\end{aligned}
$$


By the Fubini theorem, $\left(\mu \times \lambda_{\mathbb{R}_{+}^{*}}\right)(A \triangle \widetilde{B})=0$. Hence $\widetilde{B}$ is $\widetilde{\mathcal{R}}$-invariant $(\bmod 0)$. This is possible if and only if $B$ is $\mathcal{R}$-invariant. Since $\mathcal{R} \times \mathcal{R}$ is $\mu$-ergodic by the assumptions of the lemma, it follows that $\mathcal{R}$ is $\mu_{J}$-ergodic. This yields that either $\mu_{J}(B)=0$ or $\mu_{J}(X \backslash B)=0$. Hence $A$ is either $\left(\mu \times \lambda_{\mathbb{R}_{+}^{*}}\right)$-null or $\left(\mu \times \lambda_{\mathbb{R}_{+}^{*}}\right)$-conull, as desired.

In a similar way one can prove a general statement on ergodicity of cocycles on $\mathcal{R} \times \mathcal{R}$.

Lemma 9.2. Let $G$ be a locally compact second countable group. Let $\mu$ be a probability measure on $X \times X$ and let $\mu=\int_{X} \delta_{x} \times \mu^{(x)} d \kappa(x)$ be a disintegration of $\mu$ with respect to the projection $X \times X \rightarrow X$ onto the first coordinate 6 . Suppose that $\mathcal{R} \times \mathcal{R}$ is $\mu$-ergodic. Given a cocycle $\alpha: \mathcal{R} \times \mathcal{R} \rightarrow G$, we define a measurable field of cocycles $\alpha^{(x)}: \mathcal{R} \rightarrow G$ defined on $\left(X, \mu^{(x)}, \mathcal{R}\right), x \in X$, by setting $\alpha^{(x)}\left(y, y^{\prime}\right):=\alpha\left((x, y),\left(x, y^{\prime}\right)\right)$ for all $\left(y, y^{\prime}\right) \in \mathcal{R}$. If $\alpha^{(x)}$ is ergodic for $\kappa$-a.e. $x \in X$ then $\alpha$ is also ergodic.

We now compute $\mu_{J}$ and the conditional measures $\mu^{(x)}$ in the disintegration (9-2) in the case where $\mu$ is a Markov measure and $J=\{1,3,5, \ldots\}$. We also describe the measurable field of cocycles $\delta^{(x)}, x \in X$, corresponding to the cocycle $\delta$ defined in Lemma 7.3.

Lemma 9.3. Let $\mu$ be a Markov measure determined by a pair $(\lambda, \boldsymbol{P})$. Let $J$ be the set of odd positive integers. Set $\kappa_{u, v}:=\frac{Q_{u, 1} Q_{1, v}}{Q_{u, 0} Q_{0, v}}$ for $u, v=0,1$. Then

(i) $\mu_{J}$ is the Markov measure on $X$ determined by the pair $\left(\lambda, \boldsymbol{P}_{J}\right)$, where $\boldsymbol{P}_{J}=\left(P^{(2 n-1,2 n)}\right)_{n=1}^{\infty}$,

(ii) $\mu^{(x)}$ is the Bernoulli measure $\bigotimes_{n=1}^{\infty} \mu_{n}^{(x)}$ on $X$, where

$$
\mu_{n}^{(x)}(i)=\frac{P_{x_{n}, i}^{(2 n-1)} P_{i, x_{n+1}}^{(2 n)}}{P_{x_{n}, x_{n+1}}^{(2 n-1,2 n)}}, \quad i=0,1,
$$

and

(iii) $\delta^{(x)}(a, b)=\prod_{i \geq 1}\left(\kappa_{x_{i}, x_{i+1}}\right)^{b_{i}-a_{i}}$ for $(a, b) \in \mathcal{R}, a=\left(a_{i}\right)_{i=1}^{\infty}, b=\left(b_{i}\right)_{i=1}^{\infty}$, for $\mu_{J}$-a.e. $x=\left(x_{1}, x_{2}, \ldots\right) \in X$.

Proof. The first claim is obvious. To prove the second one, we take $n>0$ and a cylinder $\left[a_{1}, \ldots, a_{n}\right]_{1}^{n}$. Then we have

$$
\begin{aligned}
\mu^{(x)}\left(\left[a_{1}, \ldots, a_{n}\right]_{1}^{n}\right) & =\frac{\mu\left(\left[x_{1}, a_{1}, x_{2}, a_{2}, \ldots, x_{n}, a_{n}, x_{n+1}\right]_{1}^{2 n+1}\right)}{\mu\left(\left[x_{1}\right]_{1} \cap\left[x_{2}\right]_{3} \cap \cdots \cap\left[x_{n+1}\right]_{2 n+1}\right)} \\
& =\frac{\lambda\left(x_{1}\right) P_{x_{1}, a_{1}}^{(1)} P_{a_{1}, x_{2}}^{(2)} \cdots P_{x_{n}, a_{n}}^{(2 n-1)} P_{a_{n}, x_{n+1}}^{(2 n)}}{\lambda\left(x_{1}\right) P_{x_{1}, x_{2}}^{(1,2)} \cdots P_{x_{n}, x_{n+1}}^{(2 n-1,2 n)}} \\
& =\frac{P_{x_{1}, a_{1}}^{(1)} P_{a_{1}, x_{2}}^{(2)} \cdots \frac{P_{x_{n}, a_{n}}^{(2 n-1)} P_{a_{n}, x_{n+1}}^{(2 n)}}{P_{x_{1}, x_{2}}^{(1,2)}}}{P_{x_{n}, x_{n+1}}^{(2 n-1,2 n}}
\end{aligned}
$$

as desired. It is straightforward to verify the third claim.

\footnotetext{
${ }^{6}$ Thus the measure $\kappa$ is the projection of $\mu$ to the first coordinate and $X \ni x \mapsto \mu^{(x)}$ is the corresponding system of conditional measures on $X$.
} 
Given $x \in X$ and $n>0$, let

$$
r_{n}^{(x)}:=\frac{\mu_{n}^{(x)}(1)}{\mu_{n}^{(x)}(0)}=\frac{P_{x_{n}, 1}^{(2 n-1)} P_{1, x_{n+1}}^{(2 n)}}{P_{x_{n}, 0}^{(2 n-1)} P_{0, x_{n+1}}^{(2 n)}}
$$

Denote by $\mathcal{L}^{(x)}$ the set of limit points of the sequence $\left(r_{n}^{(x)}\right)_{n=1}^{\infty}$. Of course, if $(x, y) \in \mathcal{R}$ then $\mathcal{L}^{(x)}=\mathcal{L}^{(y)}$. Therefore the map $X \ni x \mapsto \mathcal{L}^{(x)}$ is a Borel $\mathcal{R}$ invariant map from $X$ to the space of closed subsets of the ray $[0,+\infty)$ if we endow this space with the Fell topology [Fel]. Since $\mathcal{R}$ is $\mu_{J}$-ergodic, there exists a closed subset $\mathcal{L} \subset[0,+\infty)$ such that $\mathcal{L}^{(x)}=\mathcal{L}$ for $\mu_{J}$-a.e. $x$. We say that a point $\alpha \in \mathcal{L}^{(x)}$ is good if there is an increasing sequence $n_{1}<n_{2}<\cdots$ of positive integers such that $\lim _{j \rightarrow \infty} r_{n_{j}}^{(x)}=\alpha$ and $\sum_{i>0}\left(\alpha-r_{n_{j}}^{(x)}\right)^{2}=+\infty$. If there is a segment $[\beta, \gamma] \subset[0,+\infty)$ such that the intersection $\mathcal{L} \cap[\beta, \gamma]$ is infinite then $\mathcal{L}$ contains a good point.

Theorem 9.4. Let $\mu$ be a Markov measure determined by a pair $(\lambda, \boldsymbol{P})$ and let $(X, \mu, T)$ stand for the corresponding nonsingular one-sided Markov shift.

(i) If $\sum_{u=0}^{1} \lambda(u) P_{u, v}^{(1)}=\lambda(v)$ for $v=0,1$ and $\sum_{u=0}^{1} \sum_{n=1}^{\infty}\left(P_{u, 0}^{(n)}-P_{u, 0}^{(1)}\right)^{2}<\infty$ then $(\widetilde{X}, \widetilde{\mu}, \widetilde{T})$ is isomorphic to the measure preserving two-sided shift on the probability space $(\widetilde{X}, \widetilde{\nu})$, where $\widetilde{\nu}$ is the Markov measure determined by the pair $\left(\lambda,\left(P^{(1)}\right)_{n=1}^{\infty}\right)$.

(ii) If the natural extension $(\widetilde{X}, \widetilde{\mu}, \widetilde{T})$ of $T$ is conservative and $\mathcal{L}$ contains a good non-zero point then the Maharam extension of $(\widetilde{X}, \widetilde{\mu}, \widetilde{T})$ is a weakly mixing $K$-automorphism. In particular, $(\widetilde{X}, \widetilde{\mu}, \widetilde{T})$ is of type $I I I_{1}$.

Proof. (i) follows form the final claim of Corollary 5.5.

(ii) To show that the Maharam extension of $(\widetilde{X}, \widetilde{\mu}, \widetilde{T})$ is a $K$-automorphism we will apply Theorem 7.5. For that we have to prove that the cocycle $\Delta_{S_{T}, \mu} / \delta$ is ergodic (the cocycle $\delta$ is defined in Lemma 7.3). Let $J$ stand for the set of odd positive integers. By Lemma $9.2, \Delta_{S_{T}, \mu} / \delta$ is ergodic if the cocycle $\Delta_{\mathcal{R}, \mu^{(x)}} / \delta^{(x)}$ of $\mathcal{R}$ on $\left(X, \mu^{(x)}\right)$ is ergodic for $\mu_{J}$-a.e. $x$. Given $x \in X$, partition $\mathbb{N}$ into four subsets $\mathbb{N}=J_{0,0}^{(x)} \sqcup J_{0,1}^{(x)} \sqcup J_{1,0}^{(x)} \sqcup J_{1,0}^{(x)}$, where $j \in J_{u, v}^{(x)}$ if $x_{j}=u$ and $x_{j+1}=v$. It follows from Lemma 9.3 that the quadruple $\left(X, \mu^{(x)}, \mathcal{R}, \delta^{(x)}\right)$ splits into direct product

$$
\bigotimes_{u, v=0}^{1}\left(\{0,1\}^{J_{u, v}^{(x)}}, \mu_{u, v}^{(x)}, \mathcal{R}_{u, v}^{(x)}, \delta_{u, v}^{(x)}\right)
$$

where $\mu_{u, v}^{(x)}:=\bigotimes_{i \in J_{u, v}^{(x)}} \mu_{i}^{(x)}, \mathcal{R}_{u, v}^{(x)}$ is the tail equivalence relation on the product space $\{0,1\}^{J_{u, v}^{(x)}}$ and $\delta_{u, v}^{(x)}$ is a cocycle of $\mathcal{R}_{u, v}^{(x)}$ given by

$$
\delta_{u, v}^{(x)}(a, b):=\left(\kappa_{u, v}\right)^{\sum_{i \in J_{u, v}^{(x)}}\left(b_{i}-a_{i}\right)}
$$

Hence it suffices to show that for some pair $(u, v)$, the cocycle $\Delta_{\mathcal{R}_{u, v}^{(x)}, \mu_{u, v}^{(x)}} / \delta_{u, v}^{(x)}$ is ergodic. It follows from the condition of the theorem that there is $\alpha \in \mathcal{L}^{(x)}$ and an increasing sequence $n_{1}<n_{2}<\cdots$ of positive integers such that $\alpha>$ $0, \lim _{j \rightarrow \infty} r_{n_{j}}^{(x)}=\alpha$ and $\sum_{i>0}\left(\alpha-r_{n_{j}}^{(x)}\right)^{2}=+\infty$. Passing to a subsequence, if necessary, we may assume without loss of generality that there is a pair $(u, v) \in$ $\{0,1\}^{2}$ such that $n_{j} \in J_{u, v}^{(x)}$ for all $j>0$. Then $\Delta_{\mathcal{R}_{u, v}^{(x)}, \mu_{u, v}^{(x)}} / \delta_{u, v}^{(x)}$ is ergodic in view of Remark 1.6. 


\section{Concluding Remarks And open Problems}

(1) Let $\widetilde{T}$ be the natural extension of a nonsingular one-sided Markov shift. Suppose that $\widetilde{T}$ is conservative and not of type $I I_{1}$. Is the Maharam extension of $\widetilde{T}$ a $K$-automorphism? Theorems 3.1(ii), 8.1(iii) and 9.4(ii) provide only partial answers to this question.

(2) Let $T$ and $R$ be two nonsingular one-sided Bernoulli shifts on the infinite product spaces $\left(\{0,1\}^{\mathbb{N}}, \bigotimes_{n \geq 1} \mu_{n}\right)$ and $\left(\{0,1\}^{\mathbb{N}}, \bigotimes_{n \geq 1} \nu_{n}\right)$, respectively. Suppose that the natural extensions $\widetilde{T}$ and $\widetilde{R}$ of $T$ and $R$, respectively, are conservative. Is it possible that $\mu_{1}(0) \notin\left\{\nu_{1}(0), \nu_{1}(1)\right\}$ but $\widetilde{T}$ and $\widetilde{R}$ are conjugate as nonsingular transformations? In particular, can the natural extension of an equilibrial one-sided Bernoulli shift be conjugate with the natural extension of a non-equilibrial Bernoulli shift? We note that in the probability preserving case, i.e. in the case where $\mu_{1}=\mu_{n}$ and $\nu_{1}=\nu_{n}$ for all $n>1$, if $\mu_{1}(0) \notin\left\{\nu_{1}(0), \nu_{1}(1)\right\}$ then $h(\widetilde{T}) \neq h(\widetilde{R})$ and hence $\widetilde{T}$ and $\widetilde{R}$ are not conjugate.

(3) Let $T$ be a nonsingular one-sided Markov shift on $\{0,1\}^{\mathbb{N}}$ and the corresponding Markov measure on this space is not equivalent to a Bernoulli measure. Suppose that the natural extension $\widetilde{T}$ of $T$ is conservative. Then $\widetilde{T}$ is weakly mixing. Are there nonsingular one-sided Bernoulli shifts whose natural extensions are conjugate to $\widetilde{T}$ ?

(4) What are the critical dimensions (see [DanSi] for the definition) of the natural extensions of non-equilibrial nonsingular Bernoulli shifts and bistochastic Markov shifts? Can we distinguished between equilibrial and non-equilibrial Bernoulli shifts using critical dimensions? Some estimations for these invariants were obtained in [DoMor] for the equilibrial Bernoulli case.

(5) Are there nice criteria for conservativeness of the natural extensions of the one-sided nonsingular Bernoulli and Markov shifts?

(6) We recall that given an invertible nonsingular transformation $T$ of the standard non-atomic $\sigma$-finite measure space $(X, \mu)$, the ergodic index $e(T)$ of $T$ is the smallest positive integer $d$ such that the $d$-th Cartesian power $T^{\otimes d}$ of $T$ is not ergodic. If no such integer exists, $T$ is said to be of infinite ergodic index. In a similar way one can define index of conservativeness $c(T)$ for $T$. Of course, $e(T) \leq c(T)$ (see a survey [DanSi] for more information about these indices). Suppose now that $T$ is the natural extension of a nonsingular one-sided Bernoulli shift $S$ on a product space $(Y, \nu)$. It is easy to verify that for each $d>0$, the dynamical system $\left(X^{d}, \mu^{\otimes d}, T^{\otimes d}\right)$ is the natural extension of $\left(Y^{d}, \nu^{\otimes d}, S^{\otimes d}\right)$. It is easy to see that $S^{\otimes d}$ is also exact. Hence $S^{\otimes d}$ is ergodic. Therefore, if $T^{\otimes d}$ is conservative then it is ergodic by Lemma 2.5(iii) and (i). It follows that $e(T)=c(T)$. A question arises: what are possible values of $e(T)$ when $T$ runs over the set of natural extensions of all conservative non-singular one-sided Bernoulli shifts of type $I I I_{1}$ ?

(7) Let $T$ be an ergodic conservative invertible transformation of type $I I I_{1}$. Then the Maharam extension $\widehat{T}$ of $T$ is an ergodic conservative transformation of type $I I_{\infty}$. Of course, $e(\widehat{T}) \leq e(T)$ and $c(\widehat{T}) \leq c(T)$. What are possible values of $e(\widehat{T})$ and $c(\widehat{T})$ when $T$ runs over the set of natural extensions of all conservative non-singular one-sided Bernoulli (or Markov) 
shifts? This is related to Z. Kosloff's question: does the condition $c(T)>2$ imply that $c(\widehat{T})>2$ ?

(8) How "huge" is the centralizer ${ }^{7}$ of the type $I I I_{1}$ natural extension $\widetilde{T}$ of a nonsingular one-sided Bernoulli shift $T$ ? Does the second centralizer ${ }^{8}$ of $\widetilde{T}$ consist of just the powers of $\widetilde{T}$ as in the finite measure preserving case (cf. $[\mathrm{Ru}])$ ?

(9) There are many works devoted to computation of Krieger's type and the associated flow of the tail equivalence relation $\mathcal{R}$ on the infinite product of finite spaces equipped with product measures (i.e. odometers of product type). We refer to [DanSi] for references and definition of the associated flow. It would be interesting to consider similar problems in the Markov case, for instance, in the simplest case, where $X=\{0,1\}^{\mathbb{N}}$ and $\mu$ is a nonatomic non-degenerate Markov measure determined by some pair $(\lambda, \boldsymbol{P})$. In particular, is it true that the associated flow of $(X, \mu, \mathcal{R})$ is AT (i.e. approximatively transitive in the sense of Connes and Woods [CoWo])? Some results were obtained in [Do-Qu] for the bistochastic case and in Section 6 of the present paper for the simplest "stationary" case.

(10) We note ${ }^{9}$ that the conservative infinite measure preserving $K$-automorphisms that are Maharam extensions of (the natural extensions of) type $I I I_{1}$ Bernoulli or Markov shifts do not belong to the class of conservative infinite measure preserving $K$-automorphisms introduced by Parry in [Pa]. Parry's class consists of irreducible aperiodic recurrent Markov chains with stationary transition probabilities and countable state space. Indeed, Aaronson showed in $[\mathrm{Aa}]$ that those Markov chains are non-squashable, i.e. every element from the centralizer of such a map $T$ preserves the $T$-invariant measure. In contrast, every Maharam extension is squashable.

\section{REFERENCES}

[Aa] J. Aaronson, Rational eregodicity and a metric invariant for Markov shifts, Isr. J. Math. 27 (1977), 93-123.

[ArWo] H. Araki and E. J. Woods, A classification of factors, Publ. RIMS Kyoto Univ. Ser. A 3 (1968), 51-130.

[BrDo] G. Brown and A. H. Dooley, Ergodic measures are of weak product type, Math. Proc. Cambridge Philos. Soc. 98 (1985), 129-145.

[Br-La] G. Brown, A. H. Dooley and J. Lake, On the Krieger-Araki-Woods ratio set, Tohôku Math. J. 47 (1995), 1-13.

[Ch-Pr] J. R. Choksi, J. M. Hawkins, V. S. Prasad, Abelian cocycles for nonsingular ergodic transformations and the genericity of type III 1 transformations, Monatsh. Math. 103 (1987), 187-205.

[CoWo] A. Connes and E. J. Woods, Approximately transitive flows and ITPFI factors, Ergod. Theory Dyn. Syst. 5 (1985), 203-236.

[DaHa] K. Dajani and J. Hawkins, Examples of natural extensions of nonsingular endomorphisms, Proc. Amer. Math. Soc. 120 (1994), 1211-1217.

[DanSi] A. I. Danilenko and C. E. Silva, Ergodic theory: Non-singular transformations, Mathematics of Complexity and Dynamical Systems, Springer, New York, 2012, pp. 329-356.

\footnotetext{
${ }^{7}$ The centralizer of an invertible nonsingular transformation $S$ of a probability space is the group of all nonsingular transformations of this space that commute with $S$.

${ }^{8}$ The second centralizer of an invertible nonsingular transformation $S$ of a probability space is the group of nonsingular transformations of this space that commute with every nonsingular transformation commuting with $S$.

${ }^{9}$ The authors thanks Z. Kosloff for this remark.
} 
[Do-Qu] A. N. Dooley, I. Klemes and A. N. Quas, Product and Markov measures of type III, J. Austral. Math. Soc. 64 (1998), 84-110.

[DoMor] A. N. Dooley and G. Mortiss, The critical dimensions of Hamachi shifts, Tohoku Math. J. 59 (2007), 57-66.

[FeMo] J. Feldman and C. C. Moore, Ergodic equivalence relations, cohomology and von Neumann algebras, I, Trans. Amer. Math. Soc. 234 (1977), 289-324.

[Fel] J. M. Fell, A Hausdorff topology for the closed subsets of a locally compact non-Hausdorff space, Proc. Amer. Math. Soc. 13 (1962), 472-476.

[GiSk] T. Giordano and G. Skandalis, On infinite tensor products of factors of type $I_{2}$, Ergod. Theory Dyn. Syst. 5 (1985), 565-586.

[Ha] T. Hamachi, On a Bernoulli shift with non-identical factor measures, Ergodic Th. \& Dyn. Syst. 1 (1981), 273-284.

[HaOs1] T. Hamachi and M. Osikawa, Ergodic groups of automorphisms and Krieger's theorems, Seminar on Mathematical Science of Keio Univ., vol. 3, 1981, pp. 1-113.

[HaOs2] - Computation of the associated flows of ITPF $I_{2}$ factors of type II I $I_{0}$, Geometric methods in operator algebras, Pitman Research Notes, vol. 123, Longman, New York, 1986, pp. 196-210.

[Haw] J. M. Hawkins, Amenable relations for endomorphisms, Trans. Amer. Math. Soc. 343 (1994), 169-191.

[Ka] S. Kakutani, On equivalence of infinite product measures, Ann. Math. 49 (1948), 214224 .

[Ko1] Z. Kosloff, On a type III 1 Bernoulli shift, Ergodic Theory \& Dyn. Syst. 31 (2011), $1727-1743$.

[Ko2] - On the $K$ property for Maharam extensions of Bernoulli shifts and a question of Krengel, Israel J. Math. 199 (2014), 485-506.

[Kr] U. Krengel, Transformations without finite invariant measure have strong generators, Lecture Notes in Math. 160 (1970), Springer, New York, 133-157.

[Lo] A. A. Lodkin, Absolute continuity of measures corresponding to Markov processes with discrete time, Teor. Veroyatnost. i Primenen. 16, (1971), 703-707. (Russian)

[Os] M. Osikawa, Ergodic properties of product type odometers, Lect. Notes Math. 1299 (1988), 404-414.

[Pa] W. Parry, Ergodic and spectral analysis of certain infinite measure preserving transformations, Proc. Amer. Math. Soc. 16 (1965), 960-966.

$[\mathrm{Ru}] \quad \mathrm{D}$. J. Rudolph, The second centralizer of a Bernoulli shift is just its powers, Israel J. Math. 29 (1978), 167-178.

[Sc] K. Schmidt, Cocycles on ergodic transformation groups, MacMillan lectures in mathematics, vol. 1, MacMillan (India), 1977.

[Si] C. E. Silva, On $\mu$-recurrent nonsingular endomorphisms, Isr. J. Math. 61 (1988), 1-13.

[SiEi] C. E. Silva and S. Eigen, A structure theorem for n-to-1 nonsingular endomorphisms and existence of non-recurrent measures, J. London Math. Soc. 40 (1989), 441-451.

Institute for Low Temperature Physics \& Engineering of National Academy of Sciences of Ukraine, 47 Nauky Ave., Kharkiv, 61103, UKRAine

E-mail address: alexandre.danilenko@gmail.com

Faculty of Mathematics and Computer Science, Nicolaus Copernicus University, ul. Chopina 12/18, 87-100 Toruń, Poland

E-mail address: mlem@mat.umk.pl 